\title{
Resetting of the 24-nt siRNA landscape in rice zygotes
}

2 Chenxin Li, ${ }^{1,5}$ Jonathan I. Gent, ${ }^{2,5,6}$ Hengping Xu, ${ }^{3,5}$ Hong Fu, ${ }^{3}$ Scott D. Russell,, 3 and

3 Venkatesan Sundaresan ${ }^{1,4,6}$

4

$5 \quad{ }^{1}$ Department of Plant Biology, University of California, Davis, California 95616, USA

$6 \quad 2$ Department of Plant Biology, University of Georgia, Athens, Georgia 30602, USA

$7 \quad{ }^{3}$ Department of Microbiology and Plant Biology, University of Oklahoma, Norman, Oklahoma

873019, USA

$9{ }^{4}$ Department of Plant Sciences, University of California, Davis, California 95616, USA

105 These authors contributed equally.

11 든-corresponding authors: sundar@ucdavis.edu, srussell@ou.edu, gent@uga.edu

\section{ABSTRACT}

14 Background: The zygote, a totipotent stem cell, constitutes a critical stage of the life cycle of sexually reproducing organisms. It is produced by the fusion of two differentiated cells - the egg and sperm, which in plants have radically different siRNA transcriptomes from each other and from multicellular embryos. Due to technical challenges, the epigenetic changes that accompany the zygotic transition are poorly understood.

Results: Here, we characterized the small RNA transcriptome of rice zygotes. We found widespread redistribution of 24-nt siRNAs relative to gametes, including absence of sperm

21 signature siRNAs, reduction at egg signature siRNA loci, and upregulation at seedling signature

22 siRNA loci. Loci with reduced siRNAs in zygote relative to egg were gene-distal and

23 heterochromatic, while loci with increased siRNAs relative to egg had a similar genomic

24 distribution to canonical siRNA loci. Although both egg and zygote siRNA loci had higher $\mathrm{mCHH}$ level in wildtype than in drm2 embryo, zygote but not egg siRNA loci were associated with hypermethylation in mature embryo. A small fraction of siRNA loci $(\sim 1 \%)$ called siren loci

27 accounted for $60 \%$ of all siRNAs within zygote siRNA loci, that likely arose from maternal carryover as they had similarly abundant siRNAs in egg; these siren loci were not associated with embryo hypermethylation. 
Conclusions: Taken together, our results indicate re-distribution of siRNAs in rice zygotes towards the canonical vegetative profile, that are consistent with the initiation of resetting of the gametic epigenome before the first embryonic division.

Keywords: Small RNAs, DNA methylation, Epigenome, Plant Reproduction, Zygotic genome activation

\section{Background}

Gametes and zygotes constitute critical developmental stages in the life cycle of all sexually reproducing organisms. During fertilization, the egg cell fuses with a sperm cell to form the zygote, which is an undifferentiated and totipotent stem cell that initiates embryogenesis. Flowering plants undergo double fertilization, in which a second sperm cell fuses with the central cell and gives rise to the endosperm, a nutritive tissue that nurtures the developing embryo or germinating seedling [reviewed in (Lord and Russell, 2002)]. In animals, early embryogenesis is controlled by maternal gene products pre-deposited in the egg cell. Depending on the organism, the zygotic genome does not become transcriptionally active until a number of cell divisions have occurred (Tadros and Lipshitz, 2009). Recent studies show that material-tozygote-transition in flowering plants differs markedly from most animals [reviewed in (Armenta-Medina and Gillmor, 2019)]. In rice zygotes, thousands of genes are upregulated in zygotes, many of which are undetected in the egg cell, consistent with similar observations in zygotes of maize and Arabidopsis (Chen et al., 2017; Zhao et al., 2019). Furthermore, zygotic transcription was shown to be required for early embryogenesis (Kao and Nodine, 2019; Zhao et al., 2019). These observations suggest that in angiosperms, unlike most animals, zygotes are transcriptionally active, and zygotic genome activation (ZGA) occurs in the zygote. However, similar to animals, ZGA in plants is gradual. The initial transcriptome of flowering plant zygotes is thus dominated by egg cell RNA carryover, and although newly expressed genes in the zygote are widespread and represent a significant fraction of the zygote transcriptome, their expression levels are relatively low (Anderson et al., 2017; Chen et al., 2017; Zhao et al., 2019).

Along with dynamic changes in gene expression, epigenomic reprogramming has been observed during flowering plant reproduction. In rice and maize, the egg cell is $\sim 10$ times larger than sperm in diameter, and thus 1000 times larger than the sperm cell in volume (Anderson et al., 2013; Kranz et al., 1991), and its chromatin is diffused (Scholten et al., 2002). In contrast, the 
sperm cell chromatin undergoes global condensation, paralleling animal sperm chromatin in which protamines replace histones (Kimmins and Sassone-Corsi, 2005). A male-germline specific histone $\mathrm{H} 3$ variant MGH3 (also termed H3.10) is present in the sperm cell (Borg and Berger, 2015; Okada et al., 2005), following the removal of H3.1 (Borg et al., 2020). H3.10 is resistant to trimethylation at $\mathrm{H} 3 \mathrm{~K} 27$ (H3K27me3), thus priming the activation of key genes for sperm differentiation and embryogenesis (Borg et al., 2020). Upon karyogamy, H3.10 is removed from the paternal chromatin via a replication independent process (Ingouff et al., 2007). Other histone H3 variants, such as H3.3, are also removed from egg cell chromatin upon karyogamy, followed by loading of newly-synthesized histones, again via a replication independent mechanism (Ingouff et al., 2010). In addition, other cells of both male and female gametophytes in Arabidopsis experience global chromatin changes as well. Heterochromatin is decondensed in the central cell, the cell which gives rise to endosperm (Pillot et al., 2010). A similar phenomenon occurs in the pollen vegetative cell, the cell which encapsulates the sperm cells and enables their migration through the style to the ovule (Schoft et al., 2009; Mérai et al., 2014; Hsieh et al., 2016). Relaxation of heterochromatin in the pollen vegetative cell has been reported to produce short interfering RNA (siRNA) that traffic into the sperm cells, and reinforce transposon silencing in the gametes (Slotkin et al., 2009; Calarco et al., 2011; Martínez et al., 2016; Park et al., 2016; Kim et al., 2019). Similarly, it has been proposed that siRNAs traffic from the central cell to the egg cell, as well as from the endosperm into the developing embryo (Hsieh et al., 2009; Ibarra et al., 2012; Martínez and Köhler 2017).

Concomitant with chromatin reprogramming, there is also evidence for changes in DNA methylation during plant reproduction, especially in the context of RNA-directed DNA methylation (RdDM) [reviewed in (Gehring, 2019)]. In plants, RdDM can function in both de novo and maintenance DNA methylation [reviewed in Cuerda-Gil, and Slotkin (2016)]. Briefly, 24-nt siRNAs are loaded onto an argonaute protein (AGO), which recruits the DNA methyltransferase Domains Rearranged Methyltransferase2 (DRM2). DRM2 leads to methylation in all sequence contexts, but methylation in the $\mathrm{CHH}$ context $(\mathrm{mCHH})$, where $\mathrm{H}$ is A, C or T, is a strong indicator of RdDM in both rice and maize (Tan et al., 2016, 2018; Gent et al., 2013), but not in all plants (Zemach et al., 2013). Multiple studies reported that disruption of RdDM leads to a variety of reproductive phenotypes, including aborted embryos (Autran et al., 2011; Grover et al., 2018), arrested pollen (Wang et al., 2020), defective triploid block when the 
92

seeds were produced from a 2 m maternal $\times 4$ n paternal cross (Borges et al., 2018; Erdmann et al., 2017; Martínez et al., 2018; Satyaki and Gehring, 2019) and defective floral development (Dorweiler et al., 2000; Moritoh et al., 2012). These observations suggest siRNAs and RdDM are important for normal plant reproduction.

In mammals, it has long been proposed that fusion of two epigenetically distinct gametes presents a challenge in reproduction, and resetting of the epigenome is required for the pluripotent state of the early embryo [reviewed in (Messerschmidt et al., 2014)]. Epigenome reprogramming in mammals includes large-scale erasure of somatic chromatin signatures in germ cell precursors, establishment of sex-specific signatures in the germline, and postfertilization resetting towards pluripotency [reviewed in (Messerschmidt et al., 2014; Saitou et al., 2012; Tang et al., 2016)]. The functional consequences of epigenomic changes in gametic fate acquisition and subsequent zygotic totipotency in plants are unclear. It is clear, however, that in plants, the majority of DNA methylation is stably transmitted both maternally and paternally [reviewed in (Gehring, 2019)]. In C. elegans, siRNAs can serve as carriers of transgenerational epigenetic information, in which siRNAs can be inherited across a few generations [reviewed in (Houri-Zeevi and Rechavi, 2017)]. While multiple changes in siRNA profiles have been observed during plant reproduction (Calarco et al., 2012; Grover et al., 2020; Ibarra et al., 2012; Li et al., 2020; Papareddy et al., 2020; Schoft et al., 2009; Slotkin et al., 2009), transgenerational inheritance of siRNAs, or the lack thereof, has yet to be rigorously demonstrated in plants.

In vegetative tissues such as seedlings, 24-nt siRNAs coincide with mCHH islands - short regions with high $\mathrm{CHH}$ methylation - that are enriched around genes and mark the ends of TEs and euchromatin-heterochromatin boundaries (Gent et al., 2013; Li et al., 2015). Hereafter, we refer to such a 24-nt siRNA profile as the canonical siRNA profile, since 24-nt siRNAs are the most abundant length class in most plants [reviewed in Cuerda-Gil, and Slotkin (2016)], including rice gametes ( $\mathrm{Li}$ et al., 2020, Fig 1B). We have previously shown that the siRNA transcriptome is reprogrammed in rice gametes ( $\mathrm{Li}$ et al., 2020) where siRNA transcriptomes of egg and sperm were distinct from each other in genome-wide distribution, as well as distinct from that of the seedling (Fig. 1). The relative magnitude of the egg-borne and sperm-borne contribution of siRNAs to the zygote is unknown. A recent study in Arabidopsis revealed that siRNAs from heterochromatic TEs are transiently upregulated during embryogenesis, while siRNAs from euchromatic TEs peak at mature green embryos (Papareddy et al., 2020). However, 
123

124

125

126

127

128

129

130

131

132

133

134

135

136

137

138

139

140

141

142

143

144

145

146

147

148

149

150

151

152

153

due to technical challenges due to the limiting amounts of material, currently there are no data available for siRNA transcriptomes before the preglobular embryo stage, and consequently very little is known about the siRNA landscape in plant zygotes. Since siRNA production is influenced by histone modifications and DNA methylation, and siRNAs in turn can direct histone modifications and DNA methylation (Law and Jacobsen, 2010; Matzke and Mosher, 2014; Parent et al., 2021), the siRNA transcriptome is an output and indicator of the epigenome. Given the likely importance of epigenetic reprogramming during plant reproduction and the lack of detailed studies on zygotes, we decided to characterize the small RNA transcriptome of rice zygotes to investigate changes in the small RNA transcriptome that occur soon after fertilization. The differences in genome-wide distribution of siRNAs between gametes and zygote revealed changes that indicate that the siRNA transcriptome initiates a resetting towards the canonical profile before the first cell division, concurrently with zygotic genome activation.

\section{Results}

We collected rice zygotes $\sim 9$ hours after pollination (hap), which corresponds to the completion of S-phase, just prior to the first zygotic division (Anderson et al., 2017; Ding et al., 2009). We generated small RNA transcriptomes from 6 replicates, with $\sim 50$ zygotes in each replicate. As a maternal sporophytic control, we also collected post-fertilization ovary of the same developmental stage as zygote (9 hap) and prepared small RNA transcriptomes from 3 replicates, with 10 ovaries in each replicate. For our analyses, we also included small RNA transcriptome data from rice gametes, pre-anthesis ovary ( 0 hr ovary) and seedlings (Li et al., 2020). Except where indicated otherwise, siRNAs used for analyses were small RNA reads (20nt - 25-nt) not overlapping $90 \%$ or more of their lengths with known miRNAs [miRBase v22, (Kozomara et al., 2019)], 5S rRNA, tRNA, NOR, or phasiRNA loci [as detected in Li et al., 2020], and multi-mapped reads were included in all analyses unless indicated otherwise (Fig S1A).

The global siRNA pattern in zygote is determined by siRNA transcript carryover from the egg $\underline{\text { cell, with no detectable signature of sperm cell small RNAs }}$

As we previously reported, the sperm cell has an siRNA pattern complementary to the canonical pattern of vegetative tissues, in which its 24-nt siRNAs are spread out across wide 
154 heterochromatic regions, including centromeric tandem repeats. The egg cell and ovary have a

155 pattern different from both sperm and vegetative tissues, in which 24-nt siRNAs are concentrated

156 at discrete loci (Fig 1A). We found that in a whole-genome view, the zygote had a similar

157 pattern to the egg cell (Fig 1A, zygote vs. egg track). To confirm that the similarity between

158 zygote and egg cell was not due to large numbers of residual unfertilized egg cells in the zygote

159 samples, we performed a control pollination experiment under similar conditions, and we

160 determined that 98 out of 101 pollinated rice florets produced mature seeds, implying that 3\% or

161 less of the rice florets were unfertilized (Supplemental Table 2, see Methods for additional

162 details). Thus, in our zygote samples, unfertilized egg cells might represent at most 3\% of the

163 total. We also performed differential expression analyses for miRNAs and detected 14 miRNAs

164 that were lowly expressed in all six replicates of zygote but highly expressed in ovaries of the

165 corresponding developmental stage, i.e., 9 hap (Fig S1B). Thus, the similarity between zygote

166 and ovary (Fig 1A) is unlikely to be due to small RNA contamination from ovary. A similar

167 analysis was previously used to show that the egg cell samples were also free of pre-fertilization

168 ovary contamination ( $\mathrm{Li}$ et al., 2020). 

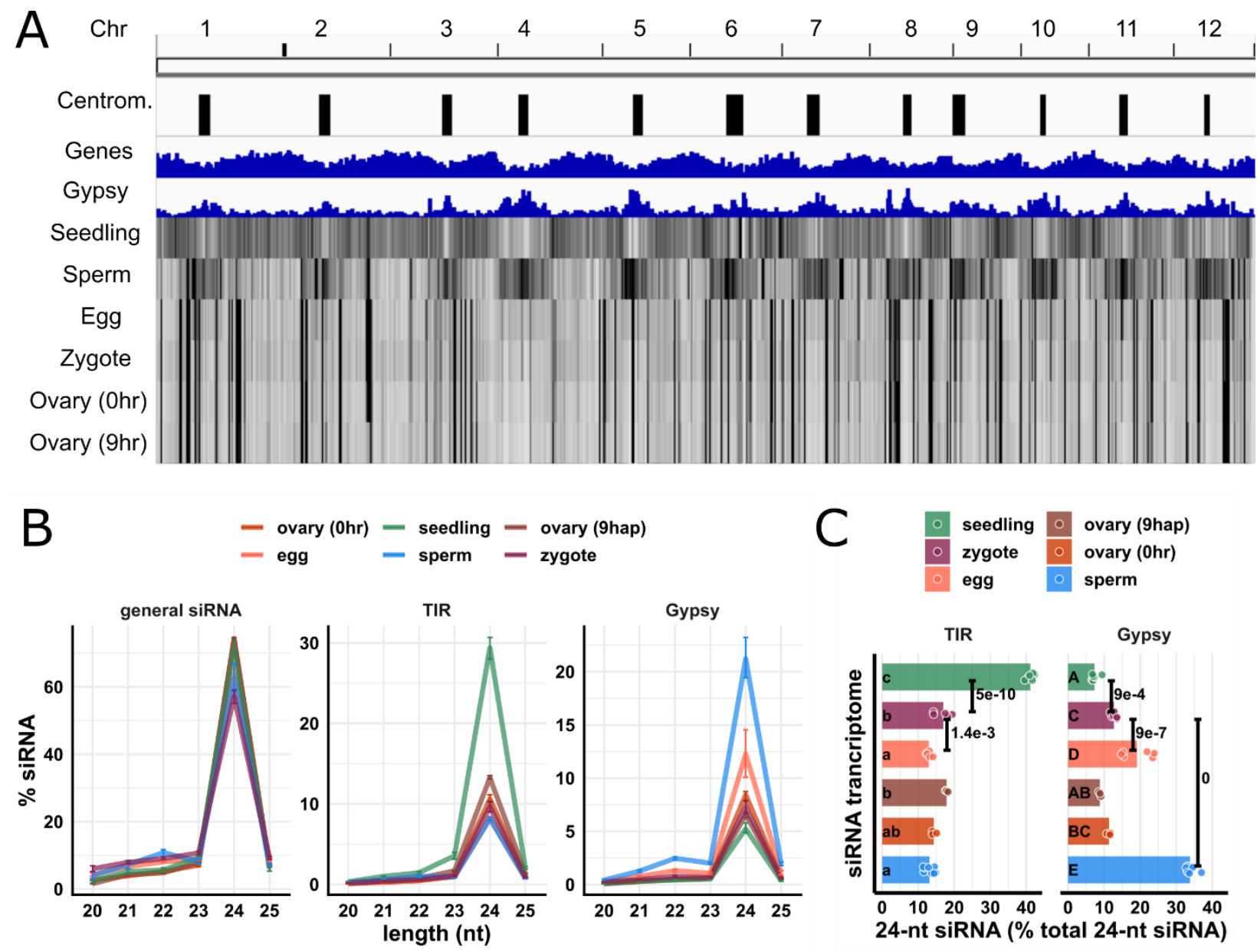

D

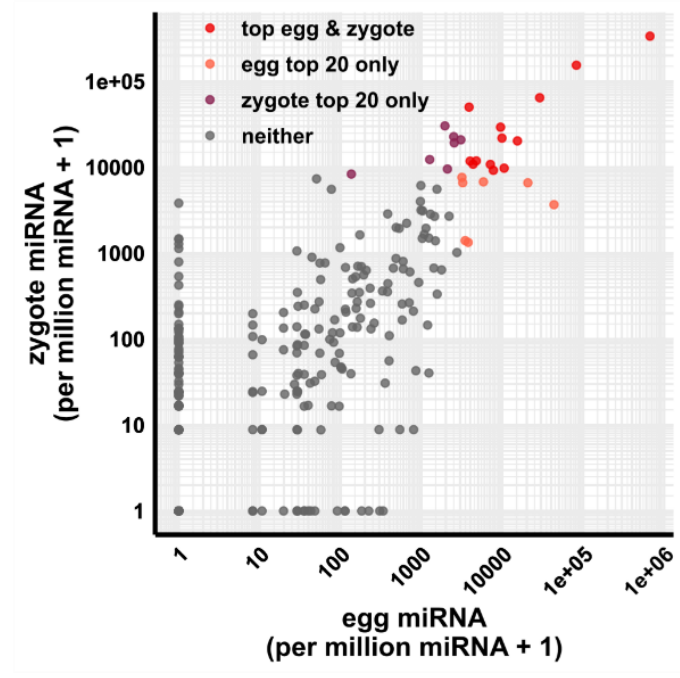

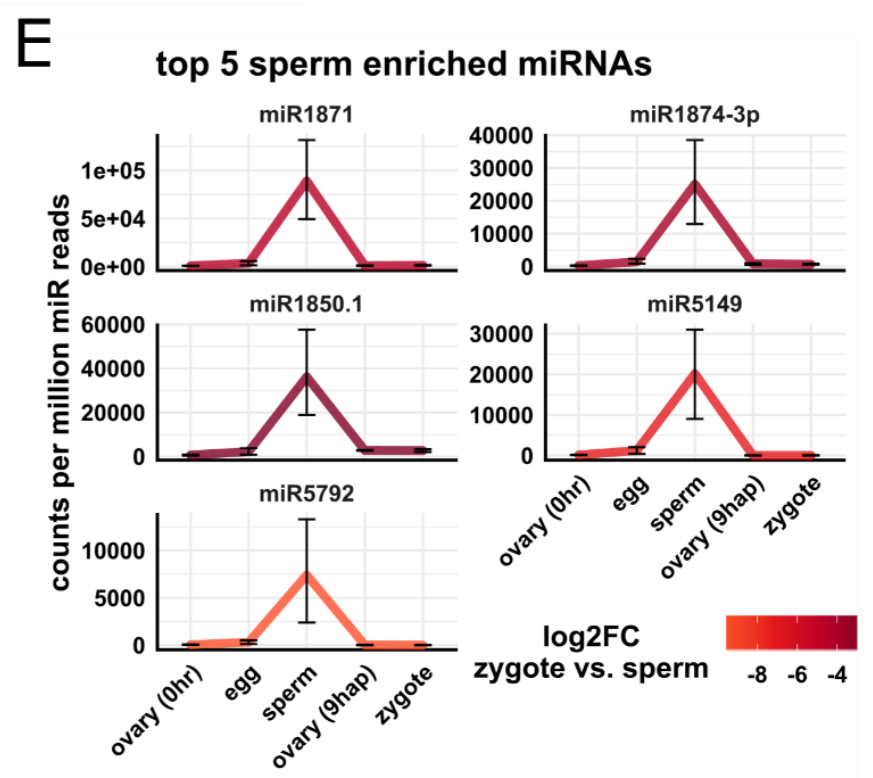

Fig 1: Overall pattern of zygote 24-nt siRNAs is similar to but not identical to egg cell. 
(A) Heat map showing abundance of 24-nt siRNA across genome at 50-kb resolution. The first three tracks are centromeres [as defined by (Mizuno et al., 2018)], genes, and Gypsy retrotransposons.

(B) Length profiles of siRNAs. y-axis values are relative to total siRNA reads $(20-25$-nt siRNAs). TIR: terminal inverted repeat transposons, CACTA superfamily excluded. Gypsy: Gypsy retrotransposons. Error bars are 95\% confidence intervals for each cell type. miRNA and phasiRNA are not included in this analysis (Fig S1A).

(C) Quantification of TIR and Gypsy panels in (B). Each data point is an siRNA transcriptome. Bar heights are averages. $\mathrm{x}$-axis values are relative to total 24-nt siRNAs. Letter grouping $(\alpha=0.05)$ and $\mathrm{P}$ values are based on Tukey tests.

(D) Scatter plot showing miRNA relative abundances in egg and zygote. Each data point is a miRNA. Axes are relative to per million miRNA reads and $\log 10$ transformed. 'top egg \& zygote' refers to intersection of the 20 highest abundant miRNAs in both egg and zygote.

(E) Top five sperm enriched miRNAs. Sperm enriched is classified as $>1000$ reads per million miRNA reads in sperm and $<500$ reads per million miRNA reads in egg. y-axis values are relative to per million miRNA reads. Color code reflects $\log 2 \mathrm{FC}$ values for zygote vs. sperm, and negative values indicate higher in sperm. Error bars are $95 \%$ confidence intervals for each cell type. See Fig S1D for additional examples. Zygote and 9 hap ovary data are from this study, all other data from Li et al., (2020).

We next looked at the length profile of siRNAs in zygotes and compared that with published data from other cell and tissue types ( $\mathrm{Li}$ et al., 2020). We found that in zygotes, as in all other tissues, 24-nt siRNAs predominated (Fig 1B). Since the abundance of siRNAs of other length classes were all relatively low, we focused on 24-nt siRNAs for further analysis. Based on relative abundance patterns, the zygote siRNAs appeared to resemble egg cell siRNAs. Like the egg cell and unlike seedling tissues, the zygote had a lower abundance of siRNAs overlapping terminal inverted repeat (TIR) transposons (PIF/Harbinger, Tc1/Mariner, Mutator, or hAT superfamilies) than seedling (Fig 1B-C, seedling vs. zygote $P=5 \mathrm{e}-10$, Tukey tests). Like the egg cell and unlike the sperm cell, the zygote had a low abundance of siRNAs overlapping Gypsy retrotransposons (Fig 1B-C, sperm vs. zygote $P=0$, Tukey tests). However, we noted that while 
202

203

204

205

206

207

208

209

210

211

212

213

214

215

216

217

218

219

220

221

222

223

224

225

226

227

228

229

230

231

232

the zygote and egg cell were similar, there were some clear differences. Zygote had significantly more siRNAs overlapping TIR elements, and significantly less siRNA overlapping Gypsy retrotransposons than the egg cell (Fig 1C, $P=1.4 \mathrm{e}-3$ and $P=9 \mathrm{e}-7$ respectively, Tukey tests).

The similarity between egg and zygote siRNA profiles can be explained by carryover from the egg cell, since the egg cell is $\sim 1000$-fold larger than the sperm cell by volume (Kranz, Bautor, and Lörz 1991; Anderson et al., 2013; Li et al., 2019). Although 24-nt siRNAs function in the nucleus, 24-nt siRNAs were found primarily in the cytoplasm of whole-plant homogenates (Ye et al., 2012). Thus, we predict that small RNAs already present in the egg cell before fertilization would contribute to much of the siRNAs present in the zygote. This is consistent with previous observations that the 50 most highly expressed genes in egg cell remained as most highly expressed in zygote, whereas the 50 most highly expressed genes in the sperm cell were low expressed in the zygote (Anderson et al., 2017, 2013). Indeed, 13 out of the 20 most abundant miRNAs in egg cells remained among the 20 most abundant miRNAs in zygote $(P=$ 3e-14, Exact test, Fig 1D). However, the miRNA accumulation patterns were not identical between zygote and egg. 32 miRNAs were detected in the zygote but not in the egg cell (> 50 reads per million miRNA reads in zygote and undetected in egg cell), and 7 miRNAs were detected in the egg cell but not in the zygote (> 50 reads per million miRNA reads in egg cell and undetected in zygote). The presence of 32 miRNAs detected in zygote but not egg cell suggests that ZGA is initiated at miRNA loci at this stage, which would be consistent with the known ZGA of other RNA polymerase II transcripts. Meanwhile, top sperm-enriched miRNAs were very much downregulated in the zygote, consistent with dilution after fertilization (Fig $\mathbf{1 E}$ and Fig S1C). Note that the expression values in the zygote were not used to define these spermenriched miRNAs, as we classified sperm-enriched miRNAs relative to egg alone. Specifically, we required >1000 reads per million miRNA reads in sperm, and $<500$ reads per million miRNA reads in the egg cell for this classification. The expression values of the full set of expressed miRNA genes [miRBase v22, (Kozomara et al., 2019)] are provided as a complementary transcriptomics resource (Supplemental Dataset 1). Taken together, these results imply that sperm small RNAs were diluted by the egg cell cytoplasm, and that much of the siRNAs detected in the zygote were due to carryover from the egg cell.

\section{$\underline{\text { Unusual features of siRNA loci with abundant siRNAs in egg, ovary, zygote, and endosperm }}$}


Zygote, like egg cell and ovary, had a very uneven siRNA distribution across the genome, where siRNAs appeared to be concentrated at discrete sites, without a clear relationship to gene density (Fig 1A). It has been previously reported that rice developing endosperm (7-8

236 days after fertilization) has a unique siRNA profile in which a small number of loci accounted

237 for the majority of siRNAs (Rodrigues et al., 2013). These siRNA loci were termed siren loci

238 (siRNA in the endosperm). A similar phenomenon was recently reported in Brassica rapa and 239 Arabidopsis ovules and endosperm (Grover et al., 2020). The term 'siren loci' was also used by

240 Grover et al to describe these loci. To further investigate this phenomenon in zygote as well as 241 egg, ovary, and endosperm, we ranked siRNA loci according to siRNA abundance in each cell 242 type (Fig 2A). In endosperm and ovaries (pre- and post-fertilization), $0.1 \%(n=73,213$ and 243102 , respectively) of the siRNA loci accounted for $60 \%$ of the total siRNA accumulation in all 244 siRNA loci for each tissue type (Fig 2A). Similarly, in egg cell and zygote, $\sim 1 \%(n=1881$ and 2451429 , respectively) of the siRNA loci accounted for $60 \%$ of the total siRNA accumulation in all 246 siRNA loci for each cell type (Fig 2A). We call these highly expressing loci siren loci,

247 independently of siRNAs in endosperm. In fact, the siren loci in rice ovaries, egg, and zygote 248 showed little correlation with the siren loci reported in rice endosperm, at least for the specific 249 endosperm stage described , i.e., 7-8 days after fertilization (Li et al., 2020; Rodrigues et al., 250 2013). Importantly, egg siren loci were stably expressed between egg and zygote, without a 251 significant decrease after fertilization (Fig 2B), which likely explains the overall similarity 252 between egg and zygote (Fig $\mathbf{1 A}$ and Fig 3E, see below). 
bioRxiv preprint doi: https://doi.org/10.1101/2020.08.31.275958; this version posted June 18, 2021. The copyright holder for this preprint (which was not certified by peer review) is the author/funder, who has granted bioRxiv a license to display the preprint in perpetuity. It is made available under aCC-BY-NC-ND 4.0 International license.

A

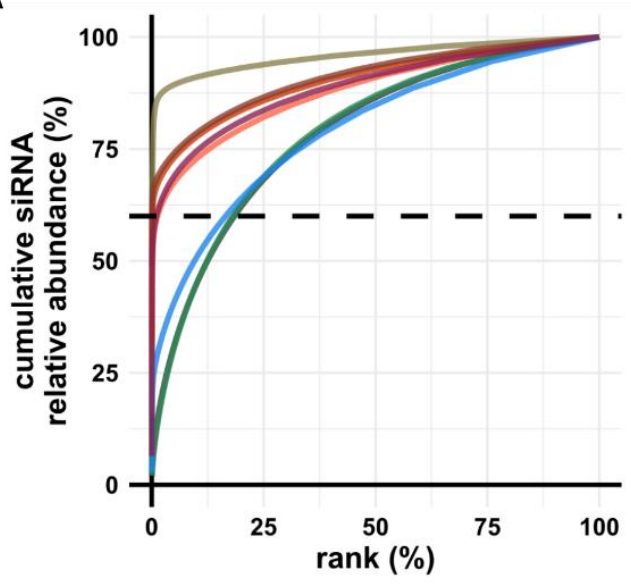

C

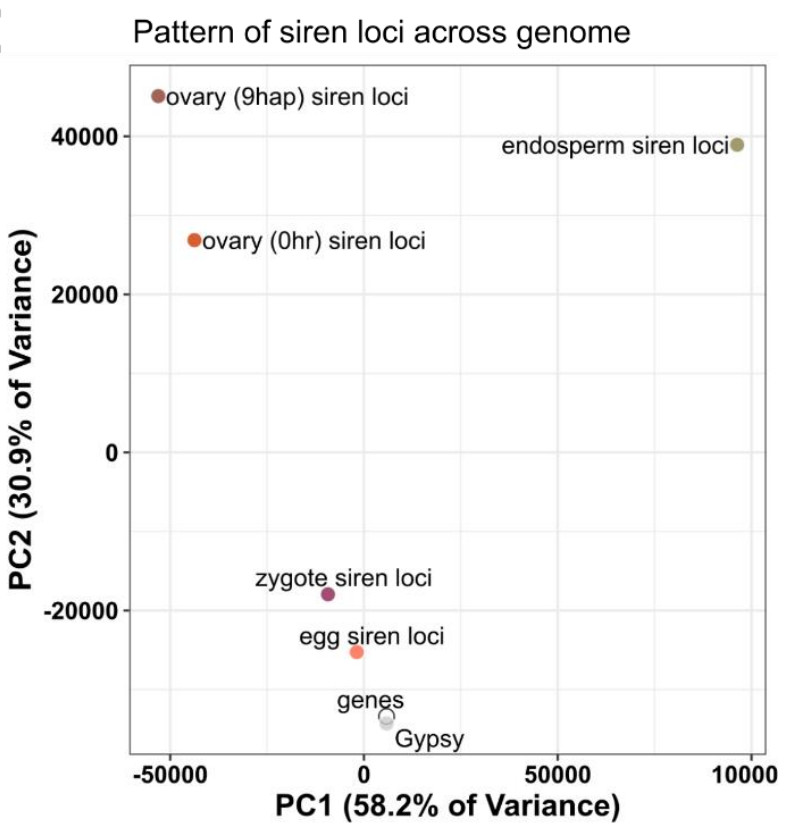

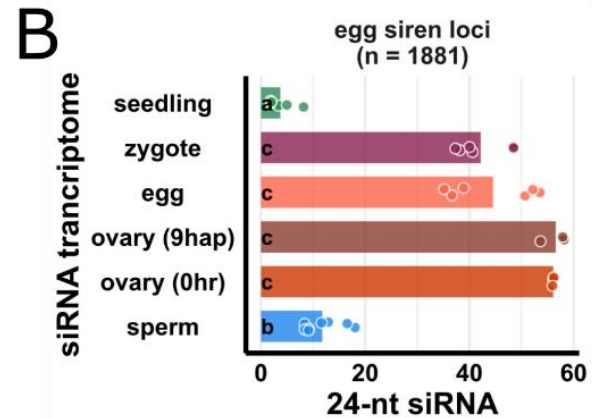

(\% total 24-nt siRNA)
D

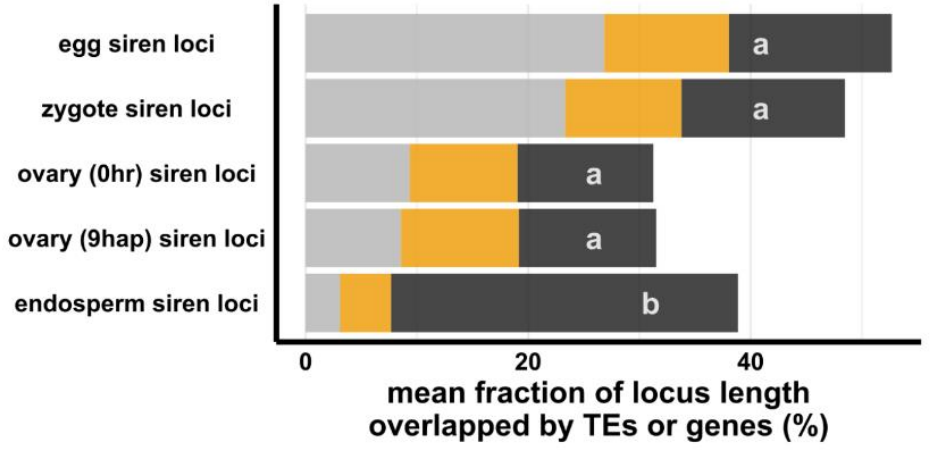

genes TIR Gypsy

E 


\section{Fig 2: Zygote siren loci are similar to siren loci detected in ovary and egg cell and stably} expressed between egg and zygote but dissimilar to siren loci detected in endosperm.

(A) $\mathrm{x}$-axis is the rank order of siRNA loci. siRNA loci with highest siRNA abundances are ranked first. $\mathrm{y}$-axis is cumulative relative abundance of siRNA in all siRNA loci. Axis values are scaled between 0 and 100\%. $0.1 \%$ of siRNA loci accounted for $60 \%$ of siRNA reads in all siRNA loci in endosperm and ovary. $1 \%$ of siRNA loci accounted for $60 \%$ of siRNA reads in all siRNA loci in egg and zygote.

(B) Bar plot showing relative abundances of 24-nt siRNA at egg siren loci. Each data point is an siRNA transcriptome. Bar heights are averages. $\mathrm{x}$-axis values are relative to total 24-nt siRNAs.

(C) Principal component plot for siren loci distribution across the genome. Distributions are evaluated at 50-kb resolution across the genome. Each data point is the distribution of a siren loci category.

(D) Stacked bar plots showing mean fraction of locus length overlapped by TEs or genes. TIR: terminal inverted repeat transposons, CACTA superfamily excluded. Gypsy: Gypsy retrotransposons.

(E) Boxplots showing 24-nt siRNA relative abundances across siren classes across cell types. Middle lines are median. Boxes span interquartile range. y-axis values are relative to per million total 24-nt siRNAs in each siRNA transcriptome. Whiskers span $2.5^{\text {th }}$ and $97.5^{\text {th }}$ percentiles.

Letter grouping ( $\alpha=0.05$ ) and $\mathrm{P}$ values are based on Tukey tests. Embryo and endosperm data from Rodrigues et al., (2013). Seedling, gametes, and pre-fertilization ovary data from Li et al., (2020).

Next, we compared the similarity among different siren loci categories based on their genomic distributions. We used principal component analysis (PCA) to cluster the genomic distributions of the loci categories based on their abundances in 50-kb windows genome-wide (Fig 2C). As reference points, the genomic distributions of genes and Gypsy retrotransposons were included. On the PC plot, endosperm siren loci were well separated from all the others along PC1, which accounts for 58\% of the variance in their genomic distributions. The rest of the siren loci categories were separated along PC2, which accounts for $31 \%$ of the variance, much 
287

288

289

290

291

292

293

294

295

296

297

298

299

300

301

302

303

304

305

306

307

308

309

310

311

312

313

314

315

316

317

less than what was explained by PC1. Ovary siren loci categories (pre- and post-fertilization) had similar genomic distributions, clustering closely together (Fig 2C). Egg and zygote siren loci also had nearly the same genomic distribution, clustering closely together (Fig 2C). All siren categories have distinct genomic distributions from distributions of genes or Gypsy elements (Fig 2C). Consistent with its unique genomic distribution, endosperm siren loci were more likely to overlap a gene (Fig 2D). On average, $~ 30 \%$ of the locus length of an endosperm siren locus was covered by a gene, whereas all the other siren categories display a similar fraction of locus length covered by genes $(\sim 13 \%, P<1.4 \mathrm{e}-4$, Tukey tests). Lastly, we compared the relative abundances of 24-nt siRNAs at different siren categories across different cell types. At endosperm siren loci, endosperm had the highest 24-nt siRNA expression, 10-fold higher than the level in embryo and more than 100-fold higher than the levels in all other cell types we examined (Fig 2E). In contrast, the other siren classes shared a siRNA accumulation pattern across cell types (Fig 2E). Ovaries (pre- and post-fertilization), egg cell and zygote all had high abundances of 24-nt siRNAs at ovary/egg/zygote siren loci, consistent with the stable expression of egg siren siRNAs in zygote (Fig 2B), while seedling, sperm, embryo, and endosperm all had low abundances of 24-nt siRNAs at these siren loci. Taken together, these distinct siRNA accumulation patterns reveal that zygote siRNAs were concentrated at discrete sites similar to egg and ovary, and that the stable expression of egg siren siRNAs explains the overall similarity between zygote and egg (Fig 1A).

Zygote gained siRNAs at canonical siRNA loci while losing siRNAs at egg-signature loci

Although the siRNA profile in the zygote was similar to the egg cell in terms of overall patterns of abundance, a deeper analysis revealed significant differences from the egg cell. We produced metagene siRNA coverage plots for seedling, gametes, and zygote, as well as pre- and post-fertilization ovaries (Fig 3A). Seedling had a strong peak upstream of the transcription start site (TSS), corresponding to where TIR transposons are enriched in the genome, with the exception of the CACTA superfamily (Han et al., 2013), and such a peak was absent in gametes and ovaries. Zygote had a significant increase in 24-nt siRNA coverage at the peak of the metagene curve relative to egg cells (Fig 3A-B, $P=3 \mathrm{e}-8$, Tukey tests). In contrast, there was no significant changes between pre- and post-fertilization ovaries (Fig 3A-B, $P=0.98$, Tukey tests). Thus, the differences between zygote and egg could not be due to trafficking of the newly- 
transcribed siRNAs from ovary. To analyze the abundance of siRNAs at individual genomic locus level, we defined siRNA loci from egg, sperm, and seedling using Shortstack (Axtell, 2013). We then classified seedling-signature loci as seedling siRNA loci that did not overlap any egg siRNA loci or sperm siRNA loci (seedling loci $\notin$ egg loci $\notin$ sperm loci, Fig 3C).

Overlapping siRNA loci were defined as at least 1-bp overlap in genomic coordinates (see also Methods). Likewise, we classified sperm-signature loci as sperm siRNA loci that did not overlap any egg or seedling siRNA loci (sperm loci $\notin$ egg loci $\notin$ seedling loci, Fig 3C), and lastly, eggsignature loci as egg siRNA loci that did not overlap any seedling or sperm siRNA loci (egg loci $\notin$ seedling loci $\notin$ sperm loci, Fig 3C). At egg-signature loci, zygote experienced a 10-fold reduction of 24-nt siRNAs (Fig 3D, $P=5 \mathrm{e}-14$, Tukey tests). At seedling-signature loci, zygote had 4.7-fold more 24-nt siRNAs than egg cell (Fig 3D, $P=1 \mathrm{e}-13$, Tukey tests). Gaining siRNAs at gene-proximal regions and seedling-signature loci is consistent with an increase of TIR siRNAs in zygote (Fig 1C). Since these seedling-signature loci did not overlap any egg siRNA loci or sperm siRNA loci, the increase of 24-nt siRNAs at seedling-signature loci in zygote was unlikely due to carryover from either gamete. At sperm-signature loci, zygote had very few 24-nt siRNAs (Fig 3D, zygote vs. sperm $P=4 \mathrm{e}-14$, Tukey tests), much like the results for miRNAs (Fig 1E), confirming small RNA contribution from sperm cell is very limited relative to egg. There was little difference in the ovaries before and after fertilization for any of these locus categories (Fig 3D, $P=0.76, P=0.84$ and $P=0.84$ at egg-, seedling- and sperm-signature loci, respectively). It is important to note that the zygote siRNA transcriptome was not used to define these locus categories. Lastly, we bioinformatically removed siren siRNAs from egg and zygote libraries (Fig 3E) and re-analyzed their genome-wide 24-nt siRNA distributions. This analysis revealed that outside of the siren loci (which as defined previously constitute $\sim 1 \%$ of all 24 -nt siRNA loci), zygote and egg were indeed distinct from each other in genome-wide 24-nt siRNA distribution (Fig 3E and Fig S2F, $P=0$, Tukey Tests). Egg cell has a slight enrichment of 24-nt siRNAs at centromeric regions, while zygote showed a relative depletion of siRNAs at centromeric regions (Fig S2G, egg vs. zygote $P=0$, Tukey tests), much like embryo (zygote vs embryo $P=0.5$, Tukey tests; Fig 3E, pink boxes indicate two examples, see also Fig S2G).

Taken together, these results indicate that the zygote has an siRNA transcriptome that is distinct from that of the egg cell, and further, that the changes from egg cell to zygote were independent of post-fertilization changes in the ovary. 
bioRxiv preprint doi: https://doi.org/10.1101/2020.08.31.275958; this version posted June 18, 2021. The copyright holder for this preprint (which was not certified by peer review) is the author/funder, who has granted bioRxiv a license to display the preprint in perpetuity. It is made available under aCC-BY-NC-ND 4.0 International license.
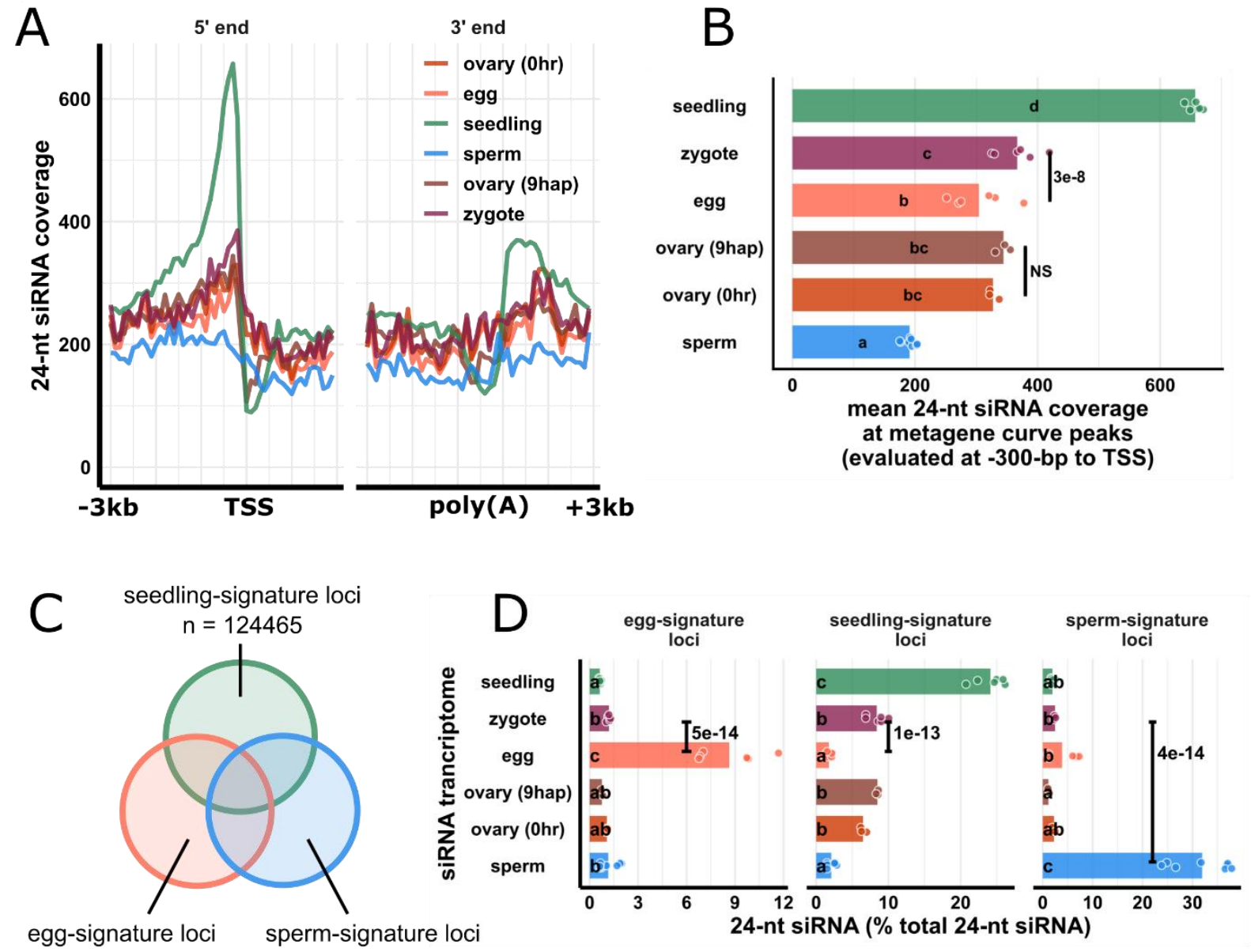

egg-signature loci

sperm-signature loci

$n=42637$

$n=146417$

E Chr

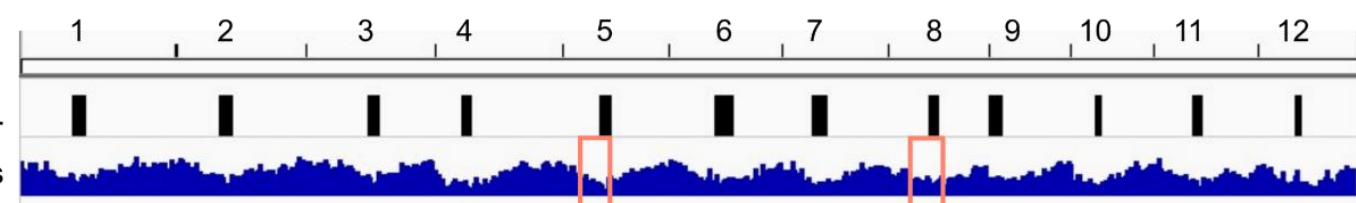

Centrom.

Genes

Gypsy

Seedling

Sperm

Egg

Zygote

Egg (-siren)

Zygote (-siren)

Embryo

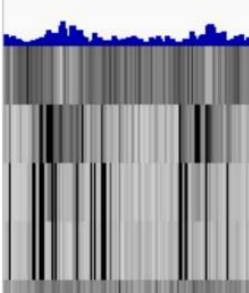

$$
\text { ) }
$$

- ovary (Ohr)

- seedling

(9hap)

$+3 k b$ 
(A) Metagene coverage plot for 24-nt siRNAs. Coverage is measured over 100-bp intervals and normalized per 1000 24-nt siRNAs. Vertical grid lines are 500-bp intervals. TSS transcription start site, poly(A) polyadenylation site.

(B) Quantification of (A) at the interval from 300 to 200-bp upstream of TSS, corresponding to the peaks of metagene curves. Each data point is an siRNA transcriptome and bar heights are averages. X-axis values are normalized per 1000 24-nt siRNAs.

(C) Venn diagram illustrating egg-signature loci (egg siRNA loci that do not overlap any seedling or sperm siRNA loci), seedling-signature loci (seedling siRNA loci that do not overlap any egg or sperm siRNA loci), and sperm-signature loci (sperm siRNA loci that do not overlap any egg or seedling siRNA loci). Sizes of overlap in Venn diagrams are not to scale.

(D) Bar plot showing relative abundances of 24-nt siRNA across siRNA loci categories defined in (C). The zygote siRNA transcriptome was not used to define these locus categories. Each data point is an siRNA transcriptome. Bar heights are averages. $\mathrm{X}$-axis values are normalized to total 24-nt siRNAs.

(E) Heat map showing abundance of 24-nt siRNA across genome at 50-kb resolution. The first three tracks are centromeres [as defined by (Mizuno et al., 2018)], genes, and Gypsy retrotransposons. '-siren' refers to siren siRNAs removed. Pink boxes highlight examples where egg and zygote are distinct.

Letter grouping ( $\alpha=0.05)$, and $\mathrm{P}$ values are based on Tukey tests. Zygote and 9 hap ovary data are from this study, embryo (7-8 DAF) from Rodrigues et al. (2013), all other data from Li et al., (2020).

To further characterize the differences between the zygote siRNA transcriptome and that of the egg cell, we next defined zygote siRNA loci using Shortstack with zygote siRNAs. We then classified Z-E loci as zygote siRNA loci that did not overlap any egg cell siRNA loci (Z loci $\notin E$ loci in set operation), E-Z loci as egg siRNA loci that did not overlap any zygote siRNA loci (E loci $\notin Z$ loci), and Z/E loci intersect as zygote siRNA loci that overlapped egg siRNA loci (Z loci $\cap$ E loci, Fig 4A). Despite the similarities between egg and zygote at the high abundance siRNA loci (Fig 1A, Fig 2B), widespread distinct siRNA loci were detected in one cell type but 
not the other. There were 101,841 E-Z loci (newly diminished siRNA loci in zygote), 94,591 Z-E loci (newly detected siRNA loci in zygote), but only 42,437 Z/E loci intersect.

When 24-nt siRNA reads at individual loci were tallied and normalized to total 24-nt siRNAs, as expected, we found that at E-Z loci, egg had 10-fold more 24-nt siRNAs than the zygote (Fig 4B, $P=0$, Tukey tests); at Z-E loci, zygote had $\sim 10$-fold more 24-nt siRNAs than egg (Fig 4B, $P=0$, Tukey tests); and no difference at $\mathrm{Z} / \mathrm{E}$ loci intersect. There were siRNAs not captured by siRNA loci. These siRNAs resided at genomic regions with insufficient siRNAs and did not meet the 0.5 RPM threshold for assignment as loci on Shortstack (see also Methods), which explains the small number of egg siRNAs at Z-E loci and the small number of zygote siRNAs at E-Z loci. There were no differences between ovaries before and after fertilization in any of the three locus categories (Fig 4B), again suggesting changes in the zygote siRNA transcriptome were not coupled with the ovary (Fig 3). In addition, the abundance of seedling siRNAs in Z-E loci and scarcity in E-Z loci revealed the emergence of a seedling-like siRNA pattern in zygote (Fig 4B). Since the seedling siRNA transcriptome was not used to classify Z-E loci, this observation suggests that the zygote has initiated a return to the canonical siRNA profile, consistent with the increase in 24-nt siRNAs from TIR transposons (Fig 1C) as well as at gene-proximal regions (Fig 3A-B) and seedling-signature loci in zygote (Fig 3D).

During ZGA of mRNA transcriptomes, genes expressed in zygote but not in egg cell all had initially low expression relative to a background of abundant maternal transcript carryover (Anderson et al., 2017; Chen et al., 2017; Zhao et al., 2019). Thus, if the siRNA transcriptome transitions similarly in the zygote, one would expect to see an initial widespread detection of low abundance 24-nt siRNAs at new loci, relative to a background of more abundant maternal carryover siRNAs corresponding to egg siren loci. Indeed, in contrast to the high abundance siRNAs of intersect loci, Z-E loci and E-Z loci overall had lower siRNA abundances than zygote/egg intersect loci (Fig 4B). Nevertheless, on average, one in every five zygote 24-nt siRNAs ( 20\%) resided at Z-E loci in the zygote. Together with the numerical abundance of Z-E loci (70\% of all zygote loci) these results suggest that newly detected siRNA loci in zygote are widespread and explain a substantial fraction of 24-nt siRNAs in zygote.

The highly expressed siren loci in egg and zygote raise the concern of whether the apparent upregulation of Z-E loci could be explained by downregulation of egg siren loci. Consistent with the stable expression of egg siren loci in zygote, including or excluding siren 
415 siRNAs did not change the results of analyses (Fig S2, Fig 3A-D, Fig 4A-B), supporting the 416 distinct distributions of non-egg-siren 24-nt siRNAs in egg and zygote (Fig 3E). Taken together,

417 changes in the zygote siRNA transcriptome are not explained by downregulation of abundance

418 egg siren siRNAs, but due to up- and downregulation of other siRNA loci that are widespread 419 across genome. 
bioRxiv preprint doi: https://doi.org/10.1101/2020.08.31.275958; this version posted June 18, 2021. The copyright holder for this preprint (which was not certified by peer review) is the author/funder, who has granted bioRxiv a license to display the preprint in perpetuity. It is made available under aCC-BY-NC-ND 4.0 International license.
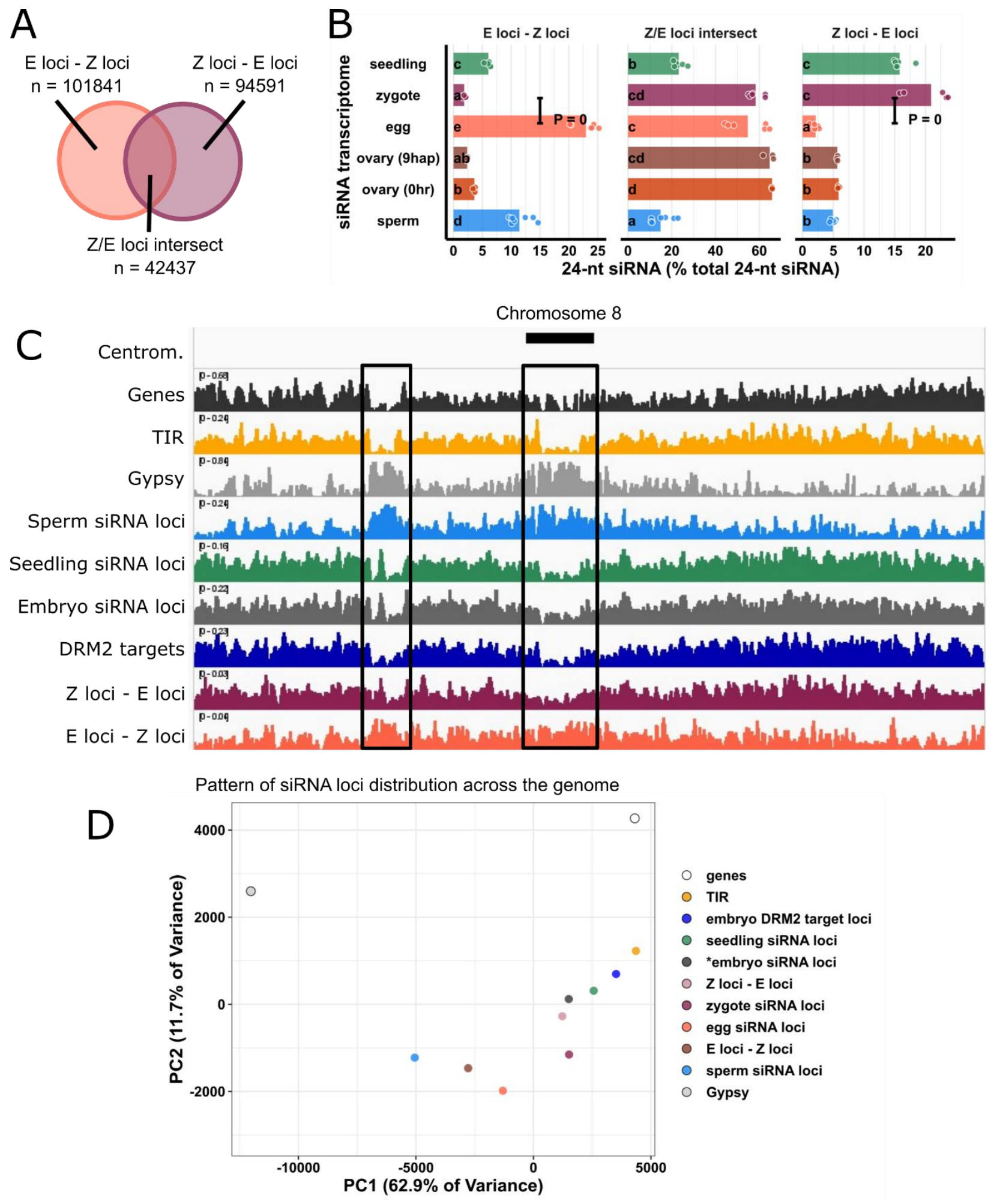

Fig 4: Widespread newly detected siRNA loci in zygote.

(A) Venn diagram illustrating E-Z loci (egg siRNA loci that do not overlap any zygote 
siRNA loci Z loci $\notin \mathrm{E}$ loci), and Z/E loci intersect (zygote siRNA loci that overlap egg siRNA loci, Z loci $\cap$ E loci). Sizes of overlap in Venn diagrams are not to scale.

(B) Quantification of 24-nt siRNA relative abundances for (A). Each data point is a siRNA transcriptome. Bar heights are averages. $\mathrm{x}$-axis-values are relative to total 24-nt siRNA reads. Letter grouping $(\alpha=0.05)$, and P values are based on Tukey tests.

(C) Distribution of siRNA loci along a chromosome. Chromosome 8 is chosen because it is one of the chromosomes with a completed sequenced centromeric region (Mizuno et al., 2018). Centrom. Centromeric regions; TIR: terminal inverted repeat transposons, CACTA superfamily excluded. Gypsy: Gypsy retrotransposons. Black boxes highlight regions with abundant Gypsy retrotransposons and relative depletion of TIR, seedling siRNA loci, embryo siRNA loci, DRM2 targets, and Z-E loci.

(D) Principal component plot showing siRNA loci distribution across the genome. Distributions are evaluated at 50-kb resolution across the genome. Each data point is the distribution of a loci category.

Zygote and 9 hap ovary data are from this study, all other data from Li et al., (2020).

Newly-detected siRNA loci in zygote resemble canonical siRNA loci in terms of genomic $\underline{\text { location and DNA methylation }}$

To investigate the patterns and characteristics of zygote siRNA loci, we compared the genomic distribution of zygote siRNA loci and Z-E loci against a set of different siRNA loci categories, including E-Z loci, egg siRNA loci, embryo siRNA loci (Rodrigues et al., 2013), seedling siRNA loci, and sperm siRNA loci. Our efforts to generate robust DNA methylome profiles for zygotes were not successful, possibly because zygotes are fragile as compared to egg cells, and the output of random-primed based methylome sequencing methods are highly sensitive to library preparation conditions ( $\mathrm{Li}$ et al., 2019). However, we had previously generated a rice $d r m 2$ mutant using CRISPR-Cas9 genome editing. By comparing $\mathrm{mCHH}$ between mature wildtype and $d r m 2$ embryos, we had previously identified a set of DRM2 targets (Li et al., 2020), which define canonical sites of RdDM. As reference points, we also included genes, TIR transposons and Gypsy retrotransposons (Kawahara et al., 2013). Z-E loci resembles the distribution of TIR, seedling and embryo siRNA loci, as well as embryo DRM2 targets. In contrast, the E-Z loci representing the newly diminished loci in zygote had a distinct pattern, 
more similar to sperm siRNA loci, which is more heterochromatic (Fig 4C). There was a relative depletion of siRNA loci from centromeric regions for zygote siRNA loci, much like canonical siRNA loci, and unlike egg siRNA loci (Fig S3A, $P=4 \mathrm{e}-10$, Tukey tests). Consistent with a more similar distribution to canonical siRNA loci, zygote siRNA loci and Z-E loci had higher degrees of overlap with seedling siRNA loci and embryo DRM2 targets, while egg siRNA and E-Z loci had low degrees of overlap, as did sperm siRNA loci (Fig S3B). Zygote siRNA loci and Z-E loci overlapped larger numbers of DRM2 targets per Mb genome space, much like seedling siRNA loci, and unlike egg siRNA loci, E-Z loci or sperm siRNA loci (Fig S3C).

To gain more information on the factors underlying the variation in siRNA loci distributions, we used principal component analysis (PCA) to cluster the genomic distributions of the above locus categories based on their abundance in 50-kb windows genome-wide (Fig 4D). The separation of locus categories along PC1 had a near-perfect rank order correlation with median distance to nearest genes (Fig S3D, rho $=-0.98, P=0$ ), and PC2 was correlated with median length of locus categories (Fig S3E, rho $=0.88, P=7 \mathrm{e}-4)$. PC1, which explained 63\% of variance in genomic distributions across loci categories, was strongly correlated with various aspects of rice genome organization. PC1 was strongly correlated with TIR transposon overlap as well as mCHH level in wildtype embryo (Fig S4A and C). PC1 was also strongly anti-correlated with Gypsy retrotransposon overlap and $\mathrm{mCG}$, and to a lesser extent $\mathrm{mCHG}$ in wildtype embryo (Fig S4B, D and E). These genomic features are mutually correlated (Fig S4F), consistent with the prior understanding of cereal genome organization (Gent et al., 2013; Han et al., 2013).

Strong correlations between PC1 (gene proximity), TE overlap, and DNA methylation led us to statistically assess the differences of these attributes among siRNA loci categories. TIR transposons, where RdDM is known to take place in cereal genomes, was gene proximal, consistent with the gene proximal distribution of embryo DRM2 targets (Fig 5A). Canonical siRNA loci, such as seedling siRNA loci and embryo siRNA loci, were closer to genes than noncanonical siRNA loci, such as sperm siRNA loci (Fig 5A). E-Z loci, the newly diminished siRNA loci in zygote, were on average much farther away from genes than Z-E loci were (Fig 5A, 2.8-kb vs. 1.6-kb, $P=0$ Tukey tests), consistent with their heterochromatic genomic distributions (Fig 4C-D, Fig S3A). Total zygote siRNA loci were closer to genes than total egg siRNA loci $(P=0)$. From egg cell to zygote, there was a $30 \%$ decrease in median distance (2.4kb vs. 1.6-kb). In contrast, from zygote to embryo (7 days after fertilization, data from Rodrigues 
486 et al., 2013), there was an 6\% decrease (1.6-kb vs. 1.5-kb). In gametes, siRNA loci were more

487 likely to overlap a Gypsy retrotransposon than a TIR transposon (Fig 5B). However, in

488 sporophytes, including zygote itself, siRNA loci are more likely to overlap a TIR transposon than

489 a Gypsy retrotransposon (Fig 5B). These observations are consistent with the results where

490 zygote had more TIR siRNAs and less Gypsy siRNAs (Fig 1C), more gene-proximal 24-nt

491 siRNAs than egg cell (Fig 3A-B), zygote had increased siRNAs in seedling-signature loci (Fig

492 3C), and seedling had comparable siRNA level with zygote in Z-E loci (Fig 4B). 
bioRxiv preprint doi: https://doi.org/10.1101/2020 08 31275958; this version posted June 18, 2021. The copyright holder for this preprint (which was not certified by peer review) is the author/funder, who has granted bioRxiv a license to display the preprint in perpetuity. It is made available under aCC-BY-NC-ND 4.0 International license.
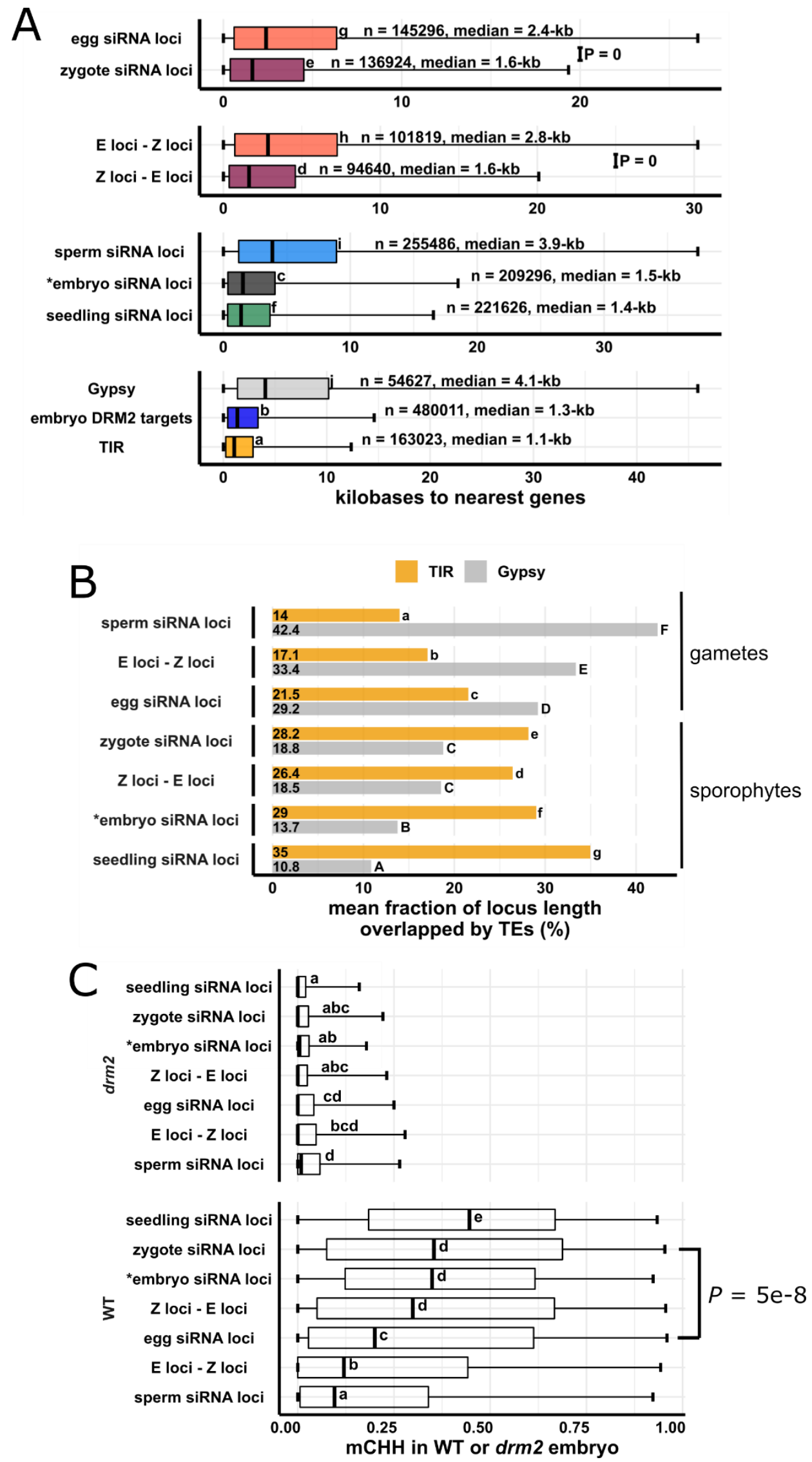


\section{Fig 5: Newly detected siRNA loci in zygote reset to the canonical siRNA profile and predict} CHH methylation in embryo in an RdDM-dependent manner.

(A) Boxplots showing distance of siRNA loci to nearest genes. Middle lines are median. Boxes span interquartile range. Whiskers span $2.5^{\text {th }}$ and $97.5^{\text {th }}$ percentiles.

(B) Bar plots showing mean locus length overlapped by TIR or Gypsy transposons across siRNA loci categories. Statistical comparisons are made across siRNA loci categories within a TE superfamily.

(C) Boxplots showing $\mathrm{CHH}$ methylation level in mature wildtype and $d r m 2$ mutant embryos. Middle lines are median. Boxes span interquartile range. Whiskers span $2.5^{\text {th }}$ and $97.5^{\text {th }}$ percentiles. E-Z loci: $n=101,841$, Z-E loci: $n=94,591$ (69\% of all zygote siRNA loci). Letter groupings $(\alpha=0.05)$ and $\mathrm{P}$ values are based on Tukey tests. *Embryo siRNA data from Rodrigues et al., (2013), which was based on a single replicate. Seedling, gametes, and prefertilization ovary data from Li et al., (2020).

Resetting to the canonical siRNA pattern might suggest that the newly detected siRNA loci in the zygote are targeted for $\mathrm{CHH}$ methylation during embryogenesis in an RdDMdependent manner. We compared DNA methylation levels across different siRNA loci categories in mature wildtype and drm2 embryos (Fig 5C, see also Fig S5). Although all siRNA loci categories were associated with RdDM during embryogenesis, as median mCHH levels were all higher in wildtype embryo than drm2 embryo, zygote siRNA loci had much higher level of $\mathrm{mCHH}$ than egg siRNA loci did in wildtype embryo (Fig 5C, $P=5 \mathrm{e}-8$ ), much like embryo and seedling siRNA loci. Importantly, newly detected siRNA loci (as represented by Z-E loci) had high $\mathrm{mCHH}$ levels in wildtype embryo $(P=0)$, whereas newly diminished siRNA loci (E-Z loci) had low $\mathrm{mCHH}$ levels in wildtype embryo. In addition, while there were smaller differences in $\mathrm{mCHG}$ and especially in $\mathrm{mCG}$ across locus categories in wildtype embryo when compared to $\mathrm{mCHH}$, the full extent of methylation across siRNA loci categories also depended on DRM2 for all three contexts (Fig S5). It is important to note that zygote siRNA loci had high degrees of overlap with canonical RdDM loci (seedling siRNA loci and embryo DRM2 targets, Fig S3B), and that zygote siRNA loci overlapped large number of DRM2 targets per Mb genome space (Fig S3C). Together with elevated mCHH level in embryo, these results suggest that newly detected zygote siRNA loci mark canonical siRNA loci that will undergo hypermethylation 
525

526

527

528

529

530

531

532

533

534

535

536

537

538

539

540

541

542

543

544

545

546

547

548

549

550

551

552

553

554

555

during embryogenesis, rather than defining hypermethylated regions that are distinct from canonical siRNA loci.

Lastly, the fact that siren loci were defined by abundant 24-nt siRNAs (Fig 2A) led us to speculate that they would have high $\mathrm{mCHH}$. In fact, we found the opposite, i.e., they had lower mCHH levels relative to other siRNA loci in each tissue (Fig S6). In ovary, siren loci had lower $\mathrm{mCHH}$ level than canonical siRNA loci (seedling siRNA loci), and ovary siRNA loci that were not siren loci had comparable mCHH level to siren loci, if not higher (Fig S6A). Similar results were found for egg cell and embryo as well (Fig S6B-C). At siren loci as well as siRNA loci that were not siren loci, DMR2 was required for $\mathrm{mCHH}$ in embryo. Lastly, both wildtype and $d r m 2$ endosperm had overall low mCHH methylation, and endosperm siren loci did not correspond to high $\mathrm{mCHH}$ level in the endosperm (Fig S6D). Unlike $\mathrm{mCHH}, \mathrm{mCG}$ and $\mathrm{mCHG}$ did not produce any notable pattern across cell types (Fig S6). Taken together, these results suggest that although the highly abundant siRNAs produced by siren loci may also target DNA methylation in a DRM2-dependent manner, they do so inefficiently as compared to siRNAs from canonical RdDM loci.

\section{Discussion}

The parental gametes have unequal contributions to the zygote siRNA transcriptome in rice

The zygote is a critical stage in the transition from gametophyte to sporophyte, but despite long standing evidence for overall chromatin reorganization after fertilization (Ingouff et al. 2010) there is little information available on the epigenetic changes in the zygotic genome that accompany this transition. We report here the first small RNA transcriptome characterization of plant zygotes. Overall, the small RNA transcriptome of the zygote is similar to that of the egg cell (Fig 1A-D), which we explain by transcript carryover from the egg cell, and the dilution of sperm cell small RNAs. This is supported by our finding that the most abundant miRNAs in egg continued to be the most abundant in zygote (Fig 1D). In contrast, top sperm-enriched miRNAs became orders of magnitudes less abundant in zygote (Fig 1E and Fig S1C), and zygote had very few 24-nt siRNAs at sperm-signature siRNA loci (Fig 3D). These observations have ensuing implications for the current models of post-fertilization silencing through the male germline. It has been proposed that sperm-transmitted siRNAs regulate TEs and balance parental contribution in the endosperm, as RdDM-mutants affect endosperms from 
2n maternal $\times 4$ paternal crosses (Borges et al., 2018; Erdmann et al., 2017; Martinez et al., 2018; Satyaki and Gehring, 2019) in Arabidopsis. Our data indicate that at least in rice embryogenesis, any effects of sperm-transmitted siRNAs on embryos are likely to be indirect. Assuming sperm-derived siRNAs are also diluted by the larger central cell, we speculate that the effect of the sperm-transmitted siRNAs may act through sperm chromatin modifications and not siRNAs themselves. In the newly formed endosperm, there is lack of an active replacement of histone variants, and sperm-derived histone variants are passively diluted through nuclear divisions (Ingouff et al., 2007, 2010). In contrast, in the zygote, histone variants are actively replaced in a replication-independent manner before the first embryonic division (Ingouff et al., 2007, 2010).

A special class of highly abundant maternal siRNAs persists in the zygote

We found that a small number of loci accounted for most of the siRNAs in egg cells and zygotes (Fig 2A). We refer to these loci as siren loci, using the term applied to similar loci in rice endosperm (Rodrigues et al., 2013), and more recently in Brassica rapa and Arabidopsis ovules (Grover et al., 2020). Importantly, egg siren siRNAs remained similarly highly expressed in the zygote (Fig 2B). Thus, upregulation of siRNAs at newly detected zygote siRNA loci cannot be explained by large downregulation of egg siren loci, which was further demonstrated by similar results for these newly detected loci obtained after excluding the siren siRNAs from the analysis (Fig S2). Siren loci were first discovered in rice endosperm (Rodrigues et al., 2013). In Arabidopsis and Brassica rapa (Grover et al., 2020), siren loci detected in ovules are also highly expressed in the endosperm; however, siren loci in rice ovary are distinct from those detected in rice endosperm (Fig 2B-E). Siren loci in the zygote were distinct from endosperm siren loci in endosperm collected 7-8 days after fertilization, instead coinciding with siren loci detected in ovary and egg cell (Fig 2B-E). However, it remains possible that the central cell and earlier stages of endosperm have an siRNA transcriptome more like that of the zygote. It has been proposed that the embryo receives siRNAs from the endosperm (Hsieh et al., 2009; Martínez and Köhler, 2017). This does not appear to be the case in 7-8 day rice seeds, since rice embryos had low siRNA abundance at endosperm siren loci at this stage (Rodrigues et al., 2013). A recent publication demonstrated that trans-acting siRNAs from ARFs (tasiR-ARF) traffic across ovule cell layers to regulate megaspore mother cell (MMC) identity in Arabidopsis (Su et al., 2020). It 
587

588

589

590

591

592

593

594

595

596

597

598

599

600

601

602

603

604

605

606

607

608

609

610

611

612

613

614

615

616

617

has also been proposed that siRNAs may traffic from the seed coat into the embryo during seed development (Grover et al., 2020, 2018). Likewise, it is possible that siren siRNAs in the egg cell and zygotes are produced in the ovary tissue instead. Although siRNAs at siren loci may direct some $\mathrm{CHH}$ methylation in ovary or during embryogenesis, they appeared to have lower mCHH level than their non-siren siRNA loci counterparts (Fig S6). Therefore, it is unlikely that siren siRNAs play a role in embryogenesis through directing DNA methylation. However, we cannot exclude the possibility that the 24-nt siRNAs of siren loci function in chromatin modification or post transcriptional silencing independently of DNA methylation in the zygote or egg, regardless of their cell type of origin.

The siRNA transcriptome landscape of rice zygotes indicates resetting towards a canonical $\underline{\text { siRNA pattern }}$

We detected widespread new zygote siRNA loci relative to the egg cell, representing $\sim 69 \%$ of all zygote siRNA loci. There were 94,591 zygote siRNA loci that did not overlap any egg siRNA loci (Z loci $\notin$ E loci or Z-E loci, Fig 4A), as compared to 42,437 siRNA loci that overlapped egg siRNA loci (Z loci $\cap \mathrm{E}$ loci or Z/E loci intersect, Fig 4A). In addition, 101,841 egg siRNA loci were diminished in zygote (E loci $\notin Z$ loci or E-Z loci, Fig 4A). In relative abundance, most of the siRNA reads were accounted for by egg siRNA carryover and stablyexpressed egg siren siRNAs (Fig 2B), and thus siRNA abundance was lower at Z-E loci than at $\mathrm{Z} / \mathrm{E}$ loci intersect which contains the siren siRNAs (Fig 4B). This low relative abundance can be understood in the context of the zygotic transition, which involves a new genomic program initiated within that one cell, so that production of new siRNAs, either by RNA polymerase IV or RNA polymerase II, will be occurring against the backdrop of egg cell RNA carryover. Similar observations have been made for zygote mRNA transcriptomes from multiple independent laboratories from different plant species (Anderson et al., 2017; Chen et al., 2017; Zhao et al., 2019), where zygote de novo expressed genes, including those with key functions in embryogenesis, were lowly expressed in the zygote. In fact, the relative abundance of the zygote siRNAs at Z-E loci ( 20\%) is similar to that of egg cell siRNAs at E-Z loci ( 22.5\%; Fig 4B), but they differ significantly in their genome-wide distribution as discussed below.

Several lines of evidence indicate the zygote has initiated a resetting towards the canonical siRNA pattern, and that such resetting is independent from the ovary. First, the zygote 
618

619

620

621

622

623

624

625

626

627

628

629

630

631

632

633

634

635

636

637

638

639

640

641

642

643

644

645

646

647

648

had increased 24-nt siRNA from TIR transposons, and decreased siRNAs from Gypsy retrotransposons, as compared to the egg cell (Fig 1C). Second, zygote had increased 24-nt siRNAs at seedling-signature loci (Fig 3D), as compared to the egg cell. In contrast, there were no significant changes to the 24-nt siRNAs at seedling-signature loci in ovaries pre- and postfertilization (Fig 3D). Moreover, seedling had comparable 24-nt siRNAs to zygote at Z-E loci

(Fig 4B). As the zygote siRNA transcriptome was not used to define seedling-signature loci, and the seedling siRNA transcriptome was not used to define Z-E loci, these results serve as an objective indication that the zygote shifted towards a more seedling-like siRNA transcriptome.

Third, zygote had increases relative to the egg cell in 24-nt siRNAs at the TSS region upstream of genes, while there was lack of a corresponding change in the ovary after fertilization (Fig 3AB). High 24-nt siRNA coverage upstream of genes around the TSS is a feature of a canonical siRNA transcriptome, as exemplified by seedling (Fig 3A). Fourth, the genomic distribution of Z-E loci is more similar to TIR transposons, embryo siRNA loci, embryo DRM2 targets and seedling siRNA loci, while that of E-Z loci is not (Fig 4C-D). Consistent with the major contribution of Z-E loci to the zygote siRNA distribution, the total set of zygote siRNA loci also displayed a closer relationship to the canonical siRNA distribution than did the total egg siRNA loci (Fig 4C). Fifth, similarities in genomic distribution were confirmed by distances to the nearest genes and TE overlaps (Fig 5A-B). Notably, there was a 30\% decrease in median distance to genes from egg to zygote, which took place over the course of less than one cell cycle. Only an 6\% decrease occurred during the transition from zygote to embryo (7 days after fertilization), occurred over the course of numerous cell cycles. Consistent with distance to nearest genes, gamete siRNA loci were more likely to overlap a Gypsy element than a TIR element; while zygote siRNA were more likely to overlap a TIR element instead, like the rest of sporophyte siRNA loci categories.

\section{Newly detected zygote siRNAs mark future $\mathrm{CHH}$ hypermethylation sites in mature embryos}

Hypermethylation of embryo has been reported in a number of angiosperm species, including Arabidopsis, soybean, chickpea, Brassica rapa, and rice (Bouyer et al., 2017;

Chakraborty et al., 2020; Kawakatsu et al., 2017; Li et al., 2020b; Lin et al., 2017; Rajkumar et al., 2020; Zhou et al., 2021). We found that newly detected siRNA loci have abundant CHH methylation in embryos that is dependent on the RdDM methyltransferase DRM2 (Fig 5C). 
Although all siRNA loci categories had higher mCHH levels in wildtype embryo than in $d r m 2$ embryo, zygote siRNA loci had higher $\mathrm{mCHH}$ levels than egg siRNA loci in mature wildtype embryo, resembling embryo and seedling siRNA loci. These results indicate that newly detected siRNA loci in zygote not only reset to canonical siRNA pattern, but also that the corresponding 24-nt siRNAs are capable of targeting high $\mathrm{CHH}$ methylation during embryogenesis. Since zygote siRNA loci have a similar distribution to canonical siRNA loci (Fig 4D) and had substantial degrees of overlap with seedling siRNA loci and embryo DRM2 targets (Fig S3B-C), zygote siRNAs are associated with high $\mathrm{CHH}$ methylation at regions similar to seedling siRNA loci and embryo DRM2 targets, instead of regions independent from canonical siRNA loci. Thus, resetting of the gametic $24 \mathrm{nt}$ siRNA loci to a distribution that results in embryo hypermethylation appears to be initiated in the zygote before the first embryonic division. Reminiscent of the increased heterochromatic siRNAs in rice gametes, a recent paper in Arabidopsis revealed that heterochromatin is decondensed during embryogenesis and promotes a transient production of siRNAs from heterochromatic TEs at least as early as the preglobular stage (Papareddy et al., 2020). The Arabidopsis embryo siRNAs from euchromatic TE and canonical siRNA loci peaked towards the end of embryo morphogenesis. However, as neither egg nor zygote siRNAs have been sequenced yet in Arabidopsis, the relationship of the heterochromatic siRNAs in Arabidopsis embryos to the siRNA reprogramming in egg cells and zygotes that is described here for rice remains to be determined.

\section{Conclusions}

Plant gametes are highly dimorphic in terms of size, chromatin (Wang and Köhler 2017; Borg and Berger 2015; Ingouff et al. 2010), and gene expression (Anderson et al., 2013), consistent with a differential reprogramming of gamete epigenomes prior to fertilization inferred from their siRNA profiles ( $\mathrm{Li}$ et al., 2020). In mammals, studies have found a progressive change in epigenomes after the two-cell embryo stage and concluded by the blastocyst stage $\mathrm{Xu}$ and Xie, 2018). Due to the extreme difficulties associated with plant zygote isolation and corresponding low-input sequencing, epigenome profiles of zygotes have remained poorly characterized in plants. The results from this study in rice suggest that while the zygote inherits maternal (but not paternal) siRNAs, the resetting to the canonical siRNA transcriptome is initiated, setting the stage for the methylation pattern in the embryo. This conclusion is consistent 
680

681

682

683

684

685

686

687

688

689

690

691

692

693

694

695

696

697

698

699

700

701

702

703

704

705

706

707

708

709

710

with previous observations in Arabidopsis that replacement of $\mathrm{H} 3$ variants occurs in the zygote before the first cell division (Ingouff et al., 2007; 2010). Lastly, as siRNA expression is influenced by histone modifications, and siRNAs can either reinforce or initiate DNA methylation and histone modifications, the siRNA transcriptome is an indicator and output of the epigenome. Thus, it appears likely that resetting of the other features of the epigenome, such as histone modifications and chromatin conformation, may also be initiated in plant zygotes after fertilization.

\section{Methods}

$\underline{\text { Plant growth condition and zygote collection }}$

Rice (Oryza sativa) variety Kitaake was grown in soil in greenhouse under natural light condition. Zygote isolation was performed as described (Anderson et al., 2017; Li et al., 2019). Briefly, rice flowers were hand pollinated. At eight to nine hours post pollination, ovaries were dissected. A transverse cut was made at the middle region of the ovary in a droplet of $0.3 \mathrm{M}$ mannitol. The lower part of the cut ovary was gently pushed using an acupuncture needle to separate selected cells under a phase contrast inverted microscope. Once the zygote was separated and floated out of the ovary incision, it was captured by a fine glass capillary and immediately frozen in liquid nitrogen. We routinely culled any unfertilized egg cells that did not conform to zygotic cell morphology during our collections (Anderson et al., 2017). 50 zygotes were collected for each replicate, and six replicates were collected. Intact ovaries at 8-9 hours after pollination were collected separately for the ovary small RNA analysis. 10 ovaries were collected for each replicate, and three replicates were collected (Supplemental Table 1).

\section{$\underline{\text { RNA extraction and small RNA library construction }}$}

RNA extractions were performed using Ambion RNAqueous Total RNA kit (AM1931), including an on-column DNase I treatment using Qiagen DNase I (79254). Total RNA was analyzed using a Bioanalyzer (Agilent) to check for RNA integrity, with the eukaryotic total RNA-pico program. RNA input for library construction was $\sim 30 \mathrm{ng}$. Small RNA libraries were made using the NEXTflex small RNA-seq kit v3 (PerkinElmer NOVA-5132-05), with the following modifications. 1/4 dilution of adapters was used. The 3' adapter ligation step was done at $20^{\circ} \mathrm{C}$ overnight. Zygote libraries were amplified at 24 cycles. Post-fertilization ovary libraries 
711

712

713

714

715

716

717

718

719

720

721

722

723

724

725

726

727

728

729

730

731

732

733

734

735

736

737

738

739

740

were amplified at 20 cycles, as pre-fertilization ovaries ( $\mathrm{Li}$ et al., 2020). The library product was size selected using PippinHT (Sage Science) 3\% agarose gel cassettes.

\section{Small RNA sequencing analysis}

Analyses were based on the Os-Nipponbare-Reference-IRGSP-1.0 reference genome (Kawahara et al., 2013). Genome annotations for transposable elements, genes, miRNAs, 5S rRNA, tRNA, NOR, CentO repeats and phasiRNA loci were performed as described (Li et al. 2020). Quality filtering, adapter trimming, PCR duplicate removal and alignment were performed as described (Li et al. 2020). Small RNA-seq reads were quality filtered and trimmed of adapters using cutadapt (Martin, 2011), parameters “-q 20 -a TGGAATTCTCGGGTGCCAAGG -e .05 -O 5 --discard-untrimmed -m 28 -M 33”. PCR duplicates were then removed using PRINSEQ, parameters "prinseq-lite.pl -fastq out_format 3 out_good -derep 1" (Schmieder and Edwards, 2011). The four random nucleotides at each end were then removed using cutadapt "-u4" followed by cutadapt "-u -4 ". Reads were aligned to the genome with BWA-backtrack (version 0.7.15) (Li and Durbin 2009), parameters "aln -t 8 -1 10." Except where indicated otherwise, multi-mapping reads were included in all analyses. The uniquely mapping subset of siRNAs was defined by having MAPQ values of at least 20 using SAMtools ( $\mathrm{Li}$ et al. 2009). Except where indicated otherwise, siRNAs used for analyses were small RNA reads (20 - 25-nt) not overlapping $90 \%$ or more of their lengths with miRNA, $5 \mathrm{~S}$ rRNA, tRNA, NOR and phasiRNA loci as determined by the BEDTools coverage tool (Quinlan and Hall, 2010). For analysis of overlaps of siRNAs at Gypsy retrotransposons, the CentO centromeric tandem repeat, Terminal Inverted Repeat (TIR) DNA transposons, and 24-nt siRNA loci, only siRNAs that overlapped by at least $50 \%$ of their lengths were counted. CACTA elements were excluded from the TIR DNA transposons. Distances to closest genes were obtained using the BEDTools closest tool. Whole-genome small RNA heat maps were made on 50-kb intervals using IGVtools (Thorvaldsdottir et al., 2013). For better visualization of midrange values, heatmap intensity was maxed out at $1.25 \times$ coverage per 10 million $24-n t$ siRNAs. 
To measure miRNA accumulation, the BEDTools coverage tool was used to count the number of $20-25$-nt reads that overlapped at least $90 \%$ of their length with annotated miRNA positions (Supplemental Dataset 1). R package EdgeR was used to analyze miRNA accumulation (McCarthy et al., 2012). Individual miRNA counts were normalized by total mapped small RNAs and filtered for $>1$ counts per million reads (CPM) in at least three libraries. Differential expression analyses were performed under $|\log 2 \mathrm{FC}|>1$ and FDR $<0.05$ cutoffs. Differential expressing miRNA genes were visualized under counts per million miRNAs.

\section{Definition of siRNA loci}

Small RNA loci were identified from the initial 20 - 25-nt total small RNA alignment BAM files using Shortstack (Axtell, 2013) after merging replicates using default parameters. Each cell type was downsampled to 3.5 million small RNAs first. For each tissue type (pre- and post-fertilization ovary, egg cell, sperm cell, zygote, seedling, embryo and endosperm), siRNA loci were defined as RPM > 0.5, 24-nt-dominant and not detected as a miRNA locus ('DicerCall=24; MIRNA=N'). Endosperm siren loci were defined as the highest expressing loci that accounted for $60 \%$ of the cumulative RPM in the endosperm. Similarly, pre- and postfertilization ovary siren loci as well as egg and zygote siren loci were defined as the highest expressing loci that accounted for $60 \%$ of the cumulative RPM in the ovary. The $60 \%$ cutoff was selected based on the turning point of cumulative expression vs. percentage rank plot of ovary (Fig 2A). Seedling-signature loci were identified as seedling siRNA loci that did not overlap any sperm siRNA loci or egg siRNA loci (seedling loci $\notin$ egg loci $\notin$ sperm loci, Fig 3C) using the BEDTools intersect tool (Quinlan and Hall, 2010). Overlaps were defined as at least 1-bp overlapping genomic coordinates. Similarly, sperm-signature loci were identified as sperm siRNA loci that did not overlap any egg siRNA loci or sperm siRNA loci (sperm loci $\notin$ egg loci $\notin$ seedling loci, Fig 3C). Egg-signature loci were identified as egg siRNA loci that did not overlap any seedling siRNA loci or sperm siRNA loci (egg loci $\notin$ seedling siRNA loci $\notin$ sperm siRNA loci, Fig 3C). Z-E loci were zygote siRNA loci that did not overlap egg siRNA loci (Z loci $\notin \mathrm{E}$ loci). E-Z loci were egg siRNA loci that did not overlap zygote siRNA loci (E loci $\notin \mathrm{Z}$ loci). Z/E loci intersect were zygote siRNA loci that overlapped egg siRNA loci (Z loci $\cap \mathrm{E}$ loci, Fig 4A). 


\section{DNA methylation analyses}

Methylation values were calculated for each locus using the mtr function of CGmapTools v0.1.2 (Guo et al., 2018) using the CGmap files generated in our previous study as input (Li et al., 2020). Only loci with more than $3(\mathrm{mC}+\mathrm{C})$ calls were included in the analyses.

$\underline{\text { Statistical analyses }}$

Tukey tests were performed using the R package emmeans (Searle et al., 1980) with multiple comparison correction using Tukey's method. Letter groupings were done at $\alpha=0.05$, where the differences between means sharing the same letter were not statistically significant. For multifactorial analyses, multiple comparisons were applied to families of tests at each interacting factor level: at the level of each TE/locus category for Fig 1C, Fig 2E, Fig 3D, Fig 4B, Fig 5B, Fig S1A, Fig S2D, Fig S2F, and Fig S3B, and at the level of genotype and context for Fig 5C, Fig S5 and Fig S6. For analyses of siRNA relative abundances or siRNA coverage across siRNA locus category across siRNA transcriptomes, a linear model was fitted using logit transformation to correct for heteroscedasticity (Fig 1C, Fig 2B, Fig 3A, Fig 3B, Fig 3D, Fig 4B, Fig S1A, Fig S2B, Fig S2D, and Fig S2F). For analyses of siRNA counts or locus counts, a linear model was fitted using $\log (\mathrm{RPM}+1)$ transformation to correct for heteroscedasticity $(\mathbf{F i g}$ 2E, Fig S2G, Fig S3A). For analyses of distances to nearest genes, a generalized linear model was fitted using log link function to correct for heteroscedasticity (Fig $\mathbf{5 A}$ ). For analyses of fraction of locus length covered by genes, a generalized linear model of quasibinomial family with logit link function was fitted to accommodate the mean-error relationship of fractional data (Fig 2D, FigS3B, Fig 5B). For analyses of DNA methylation levels across different locus categories, a generalized linear model of quasibinomial family with logit link function was fitted to accommodate the mean-error relationship of proportion data (Fig 5C, Fig S5, Fig S6). For analysis of correlations between PC1 (Fig 4D), distance to nearest genes, TE overlaps and DNA methylation, Spearman's rank order correlation was used (Fig S3D-E, Fig S4). P values $<2.2 \mathrm{e}-$ 16, which is the smallest positive floating point number $\mathrm{R}$ can display ( $\mathrm{R}$ Core Team 2020), were treated as 0 by $R$, and reported as such in this study.

\section{Supplemental information}

Additional file 1: Table S1. General mapping statistics 
Additional file 2: Table S2. Pollination success rates

Additional file 3: Supplemental figures. Fig S1-S6

Additional file 4: Supplemental Dataset1. miRNA read counts

\section{Declarations}

\section{Acknowledgements}

We thank Zachary Liechty and Christian Santos for assistance in R programming; and Alina Yalda, Jake Anichowski, and Michelle Binyu Cui for greenhouse maintenance and technical assistance. The UC Davis Genome Center provided Illumina sequencing, library quality control and size selection services. CL also acknowledges partial support by Elsie Taylor Stocking Memorial Fellowship from the Department of Plant Biology at University of California, Davis. This study was supported in part by resources and technical expertise from the Georgia Advanced Computing Resource Center, a partnership between the University of Georgia's Office of the Vice President for Research and Office of the Vice President for Information Technology.

\section{Availability of data and materials}

All small RNA data have been deposited in the Sequence Read Archive, BioProject

PRJNA533115. All R codes regarding data visualization and statistical analyses were deposited in https://github.com/cxli233/zygote smRNA/

\section{Author contributions}

CL, JIG, SDR and VS designed the study. HX and HF collected zygotes. SDR supervised zygote collections. CL produced small RNA sequencing libraries. CL and JIG analyzed data. VS supervised data collection and analyses. CL wrote the manuscript with input from all authors.

\section{Funding}

This research was funded by the National Science Foundation (IOS-1547760) and the U.S.

Department of Agriculture (USDA) Agricultural Experiment Station (CA-D-XXX-6973-H).

\section{Ethics approval and consent to participate}

Not applicable 


\section{Consent for publication}

836 Not application

\section{Competing interests}

839 The authors declare that they have no conflicts of interests.

840

\section{References}

Anderson, S.N., Johnson, C.S., Chesnut, J., Jones, D.S., Khanday, I., Woodhouse, M., Li, C., Conrad, L.J., Russell, S.D., Sundaresan, V., 2017. The Zygotic Transition Is Initiated in Unicellular Plant Zygotes with Asymmetric Activation of Parental Genomes. Dev. Cell 43, 349-358.e4. https://doi.org/10.1016/j.devcel.2017.10.005

Anderson, S.N., Johnson, C.S., Jones, D.S., Conrad, L.J., Gou, X., Russell, S.D., Sundaresan, V., 2013. Transcriptomes of isolated Oryza sativa gametes characterized by deep sequencing: evidence for distinct sex-dependent chromatin and epigenetic states before fertilization. Plant J. 76, 729741. https://doi.org/10.1111/tpj.12336

Armenta-Medina, A., Gillmor, C.S., 2019. Genetic, molecular and parent-of-origin regulation of early embryogenesis in flowering plants, in: Current Topics in Developmental Biology. Elsevier, pp. 497-543. https://doi.org/10.1016/bs.ctdb.2018.11.008

Autran, D., Baroux, C., Raissig, M.T., Lenormand, T., Wittig, M., Grob, S., Steimer, A., Barann, M., Klostermeier, U.C., Leblanc, O., Vielle-Calzada, J.-P., Rosenstiel, P., Grimanelli, D., Grossniklaus, U., 2011. Maternal Epigenetic Pathways Control Parental Contributions to Arabidopsis Early Embryogenesis. Cell 145, 707-719. https://doi.org/10.1016/j.cell.2011.04.014

Axtell, M.J., 2013a. ShortStack: Comprehensive annotation and quantification of small RNA genes. RNA 19, 740-751. https://doi.org/10.1261/rna.035279.112

Axtell, M.J., 2013b. ShortStack: Comprehensive annotation and quantification of small RNA genes. RNA 19, 740-751. https://doi.org/10.1261/rna.035279.112

Borg, M., Berger, F., 2015. Chromatin remodelling during male gametophyte development. Plant J. 83, 177-188. https://doi.org/10.1111/tpj.12856

Borg, M., Jacob, Y., Susaki, D., LeBlanc, C., Buendía, D., Axelsson, E., Kawashima, T., Voigt, P., Boavida, L., Becker, J., Higashiyama, T., Martienssen, R., Berger, F., 2020. Targeted reprogramming of H3K27me3 resets epigenetic memory in plant paternal chromatin. Nat. Cell Biol. 22, 621-629. https://doi.org/10.1038/s41556-020-0515-y

Borges, F., Parent, J., Ex, F. Van, Wolff, P., Martínez, G., Köhler, C., Martienssen, R.A., 2018. Transposonderived small RNAs triggered by miR845 mediate genome dosage response in Arabidopsis. Nat. Genet. 50. https://doi.org/10.1038/s41588-017-0032-5

Bouyer, D., Kramdi, A., Kassam, M., Heese, M., Schnittger, A., Roudier, F., Colot, V., 2017. DNA methylation dynamics during early plant life. Genome Biol. 18, 179. https://doi.org/10.1186/s13059-017-1313-0

Calarco, J.P., Borges, F., Donoghue, M.T.A., Ex, F. Van, Jullien, P.E., Lopes, T., Gardner, R., Berger, F., Feijo, A., Becker, D., Martienssen, R.A., 2011. Reprogramming of DNA Methylation in Pollen Guides Epigenetic Inheritance via Small RNA. Cell. https://doi.org/10.1016/j.cell.2012.09.001 
Calarco, J.P., Borges, F., Donoghue, M.T.A., Van Ex, F., Jullien, P.E., Lopes, T., Gardner, R., Berger, F., Feijó, J.A., Becker, J.D., Martienssen, R.A., 2012. Reprogramming of DNA Methylation in Pollen Guides Epigenetic Inheritance via Small RNA. Cell 151, 194-205. https://doi.org/10.1016/j.cell.2012.09.001

Chakraborty, T., Kendall, T., Grover, J.W., Mosher, R.A., 2020. Embryo CHH hypermethylation is mediated by RdDM and is autonomously directed in Brassica rapa (preprint). Plant Biology. https://doi.org/10.1101/2020.08.26.268573

Chen, J., Strieder, N., Krohn, N.G., Cyprys, P., Sprunck, S., Engelmann, J.C., Dresselhaus, T., 2017. Zygotic Genome Activation Occurs Shortly after Fertilization in Maize. Plant Cell 29, 2106-2125. https://doi.org/10.1105/tpc.17.00099

Ding, J., Shen, J., Li, W., Yang, H., 2009. Cytological Observation of Double Fertilization and Its Duration in Oryza sativa. Chi N Bull Bot 473-483.

Dna, N.R., Cuerda-gil, D., Slotkin, R.K., 2016. Non-canonical RNA-directed DNA methylation. https://doi.org/10.1038/NPLANTS.2016.163

Dorweiler, J.E., Carey, C.C., Kubo, K.M., Hollick, J.B., Kermicle, J.L., Chandler, V.L., 2000. mediator of paramutation1 Is Required for Establishment and Maintenance of Paramutation at Multiple Maize Loci 19.

Erdmann, R.M., Satyaki, P.R. V, Klosinska, M., Gehring, M., Erdmann, R.M., Satyaki, P.R. V, Klosinska, M., Gehring, M., 2017. A Small RNA Pathway Mediates Allelic Dosage in A Small RNA Pathway Mediates Allelic Dosage in Endosperm. CellReports 21, 3364-3372. https://doi.org/10.1016/j.celrep.2017.11.078

Gehring, M., 2019. Epigenetic dynamics during flowering plant reproduction: evidence for reprogramming? New Phytol. 224, 91-96. https://doi.org/10.1111/nph.15856

Gent, Jonathan I, Ellis, N.A., Guo, L., Harkess, A.E., Yao, Y., Zhang, X., Dawe, R.K., 2013. CHH islands : de novo DNA methylation in near-gene chromatin regulation in maize 628-637. https://doi.org/10.1101/gr.146985.112.as

Gent, J. I., Ellis, N.A., Guo, L., Harkess, A.E., Yao, Y., Zhang, X., Dawe, R.K., 2013. CHH islands: de novo DNA methylation in near-gene chromatin regulation in maize. Genome Res. 23, 628-637. https://doi.org/10.1101/gr.146985.112

Grover, J.W., Burgess, D., Kendall, T., Baten, A., Pokhrel, S., King, G.J., Meyers, B.C., Freeling, M., Mosher, R.A., 2020. Abundant expression of maternal siRNAs is a conserved feature of seed development. Proc. Natl. Acad. Sci. 202001332. https://doi.org/10.1073/pnas.2001332117

Grover, J.W., Kendall, T., Baten, A., Burgess, D., Freeling, M., King, G.J., Mosher, R.A., 2018. Maternal components of RNA -directed DNA methylation are required for seed development in Brassica rapa. Plant J. 94, 575-582. https://doi.org/10.1111/tpj.13910

Guo, W., Zhu, P., Pellegrini, M., Zhang, M.Q., Wang, X., Ni, Z., 2018. CGmapTools improves the precision of heterozygous SNV calls and supports allele-specific methylation detection and visualization in bisulfite-sequencing data. Bioinformatics 34, 381-387. https://doi.org/10.1093/bioinformatics/btx595

Han, Y., Qin, S., Wessler, S.R., 2013. Comparison of class 2 transposable elements at superfamily resolution reveals conserved and distinct features in cereal grass genomes. BMC Genomics 14, 71. https://doi.org/10.1186/1471-2164-14-71

Houri-Zeevi, L., Rechavi, O., 2017. A Matter of Time: Small RNAs Regulate the Duration of Epigenetic Inheritance. Trends Genet. 33, 46-57. https://doi.org/10.1016/j.tig.2016.11.001

Hsieh, P., He, S., Buttress, T., Gao, H., Couchman, M., Fischer, R.L., 2016. Arabidopsis male sexual lineage exhibits more robust maintenance of CG methylation than somatic tissues. PNAS 113. https://doi.org/10.1073/pnas.1619074114 
Hsieh, T.-F., Ibarra, C.A., Silva, P., Zemach, A., Eshed-Williams, L., Fischer, R.L., Zilberman, D., 2009. Genome-Wide Demethylation of Arabidopsis Endosperm. Science 324, 1451-1454. https://doi.org/10.1126/science.1172417

Ibarra, C.A., Feng, X., Schoft, V.K., Hsieh, T.-F., Uzawa, R., Rodrigues, J.A., Zemach, A., Chumak, N., Machlicova, A., Nishimura, T., Rojas, D., Fischer, R.L., Tamaru, H., Zilberman, D., 2012. Active DNA Demethylation in Plant Companion Cells Reinforces Transposon Methylation in Gametes. Science 337, 1360-1364. https://doi.org/10.1126/science.1224839

Ingouff, M., Hamamura, Y., Gourgues, M., Higashiyama, T., 2007. Distinct Dynamics of HISTONE3 Variants between the Two Fertilization Products in Plants. Curr. Biol. 1032-1037. https://doi.org/10.1016/j.cub.2007.05.019

Ingouff, M., Rademacher, S., Holec, S., Xin, N., Readshaw, A., Foo, S.H., 2010. Report Zygotic Resetting of the HISTONE 3 Variant Repertoire Participates in Epigenetic Reprogramming in Arabidopsis. Curr. Biol. 2137-2143. https://doi.org/10.1016/j.cub.2010.11.012

Kao, P., Nodine, M.D., 2019. Transcriptional Activation of Arabidopsis Zygotes Is Required for Initial Cell Divisions. Sci. Rep. 9, 17159. https://doi.org/10.1038/s41598-019-53704-2

Kawahara, Y., de la Bastide, M., Hamilton, J.P., Kanamori, H., McCombie, W.R., Ouyang, S., Schwartz, D.C., Tanaka, T., Wu, J., Zhou, S., Childs, K.L., Davidson, R.M., Lin, H., Quesada-Ocampo, L., Vaillancourt, B., Sakai, H., Lee, S.S., Kim, J., Numa, H., Itoh, T., Buell, C.R., Matsumoto, T., 2013a. Improvement of the Oryza sativa Nipponbare reference genome using next generation sequence and optical map data. Rice 6, 4. https://doi.org/10.1186/1939-8433-6-4

Kawahara, Y., de la Bastide, M., Hamilton, J.P., Kanamori, H., McCombie, W.R., Ouyang, S., Schwartz, D.C., Tanaka, T., Wu, J., Zhou, S., Childs, K.L., Davidson, R.M., Lin, H., Quesada-Ocampo, L., Vaillancourt, B., Sakai, H., Lee, S.S., Kim, J., Numa, H., Itoh, T., Buell, C.R., Matsumoto, T., 2013b. Improvement of the Oryza sativa Nipponbare reference genome using next generation sequence and optical map data. Rice 6, 4. https://doi.org/10.1186/1939-8433-6-4

Kawakatsu, T., Nery, J.R., Castanon, R., Ecker, J.R., 2017. Dynamic DNA methylation reconfiguration during seed development and germination. Genome Biol. 18, 171. https://doi.org/10.1186/s13059-017-1251-x

Kim, M.Y., Ono, A., Scholten, S., Kinoshita, T., Zilberman, D., Okamoto, T., Fischer, R.L., 2019. DNA demethylation by ROS1a in rice vegetative cells promotes methylation in sperm. Proc. Natl. Acad. Sci. 116, 9652-9657. https://doi.org/10.1073/pnas.1821435116

Kimmins, S., Sassone-corsi, P., 2005. Chromatin remodelling and epigenetic features of germ cells. Nature 583-589.

Kozomara, A., Birgaoanu, M., Griffiths-Jones, S., 2019a. miRBase: from microRNA sequences to function. Nucleic Acids Res. 47, D155-D162. https://doi.org/10.1093/nar/gky1141

Kozomara, A., Birgaoanu, M., Griffiths-Jones, S., 2019b. miRBase: from microRNA sequences to function. Nucleic Acids Res. 47, D155-D162. https://doi.org/10.1093/nar/gky1141

Kranz, E., Bautor, J., Lörz, H., 1991. In vitro fertilization of single, isolated gametes of maize mediated by electrofusion. Sex. Plant Reprod. 4. https://doi.org/10.1007/BF00194565

Law, J.A., Jacobsen, S.E., 2010. Establishing, maintaining and modifying DNA methylation patterns in plants and animals. Nat. Rev. Genet. 11, 204-220. https://doi.org/10.1038/nrg2719

Li, C., Xu, H., Fu, F.-F., Russell, S.D., Sundaresan, V., Gent, J.I., 2020a. Genome-wide redistribution of 24nt siRNAs in rice gametes. Genome Res. 30, 173-184. https://doi.org/10.1101/gr.253674.119

Li, C., Xu, H., Fu, F.-F., Russell, S.D., Sundaresan, V., Gent, J.I., 2020b. Genome-wide redistribution of 24nt siRNAs in rice gametes. Genome Res. 30, 173-184. https://doi.org/10.1101/gr.253674.119

Li, C., Xu, H., Russell, S.D., Sundaresan, V., 2019. Step-by-step protocols for rice gamete isolation. Plant Reprod. 32, 5-13. https://doi.org/10.1007/s00497-019-00363-y 
1000

1001

1002

1003

1004

1005

1006

1007

1008

1009

1010

1011

1012

1013

1014

1015

1016

Li, H., Durbin, R., 2009. Fast and accurate short read alignment with Burrows-Wheeler transform. Bioinformatics 25, 1754-1760. https://doi.org/10.1093/bioinformatics/btp324

Li, H., Handsaker, B., Wysoker, A., Fennell, T., Ruan, J., Homer, N., Marth, G., Abecasis, G., Durbin, R., 1000 Genome Project Data Processing Subgroup, 2009. The Sequence Alignment/Map format and SAMtools. Bioinformatics 25, 2078-2079. https://doi.org/10.1093/bioinformatics/btp352

Li, Q., Gent, J.I., Zynda, G., Song, J., Makarevitch, I., Hirsch, C.D., Hirsch, C.N., Dawe, R.K., Madzima, T.F., McGinnis, K.M., Lisch, D., Schmitz, R.J., Vaughn, M.W., Springer, N.M., 2015. RNA-directed DNA methylation enforces boundaries between heterochromatin and euchromatin in the maize genome. Proc. Natl. Acad. Sci. 112, 14728-14733. https://doi.org/10.1073/pnas.1514680112

Li, X., Chen, L., Zhang, Q., Sun, Y., Li, Q., Yan, J., 2019. BRIF-Seq: Bisulfite-Converted Randomly Integrated Fragments Sequencing at the Single-Cell Level. Mol. Plant 12, 438-446. https://doi.org/10.1016/j.molp.2019.01.004

Lin, J.-Y., Le, B.H., Chen, M., Henry, K.F., Hur, J., Hsieh, T.-F., Chen, P.-Y., Pelletier, J.M., Pellegrini, M., Fischer, R.L., Harada, J.J., Goldberg, R.B., 2017. Similarity between soybean and Arabidopsis seed methylomes and loss of non-CG methylation does not affect seed development. Proc. Natl. Acad. Sci. 114, E9730-E9739. https://doi.org/10.1073/pnas.1716758114

Lord, E.M., Russell, S.D., 2002. The Mechanisms of Pollination and Fertilization in Plants. Annu. Rev. Cell Dev. Biol. 18, 81-105. https://doi.org/10.1146/annurev.cellbio.18.012502.083438

Martin, M., 2011. Cutadapt Removes Adapter Sequences From High-Throughput Sequencing Reads. EMBnetjournal 17, 3.

Martinez, G., Köhler, C., 2017. Role of small RNAs in epigenetic reprogramming during plant sexual reproduction. Curr. Opin. Plant Biol. 36, 22-28. https://doi.org/10.1016/j.pbi.2016.12.006

Martínez, G., Panda, K., Köhler, C., Slotkin, R.K., 2016. Silencing in sperm cells is directed by RNA movement from the surrounding nurse cell. Nat. Plants 2, 1-8. https://doi.org/10.1038/nplants.2016.30

Martinez, G., Wolff, P., Wang, Z., Moreno-romero, J., Santos-gonzález, J., Conze, L.L., Defraia, C., Slotkin, R.K., Köhler, C., 2018. Paternal easiRNAs regulate parental genome dosage in Arabidopsis. Nat. Genet. 50. https://doi.org/10.1038/s41588-017-0033-4

Matzke, M.A., Mosher, R.A., 2014. RNA-directed DNA methylation: an epigenetic pathway of increasing complexity. Nat. Rev. Genet. 15, 394-408. https://doi.org/10.1038/nrg3683

McCarthy, D.J., Chen, Y., Smyth, G.K., 2012. Differential expression analysis of multifactor RNA-Seq experiments with respect to biological variation. Nucleic Acids Res. 40, 4288-4297. https://doi.org/10.1093/nar/gks042

Mérai, Z., Chumak, N., García-Aguilar, M., Hsieh, T.-F., Nishimura, T., Schoft, V.K., Bindics, J., Ślusarz, L., Arnoux, S., Opravil, S., Mechtler, K., Zilberman, D., Fischer, R.L., Tamaru, H., 2014. The AAAATPase molecular chaperone Cdc48/p97 disassembles sumoylated centromeres, decondenses heterochromatin, and activates ribosomal RNA genes. Proc. Natl. Acad. Sci. 111, 16166-16171. https://doi.org/10.1073/pnas.1418564111

Messerschmidt, D.M., Knowles, B.B., Solter, D., 2014. DNA methylation dynamics during epigenetic reprogramming in the germline and preimplantation embryos. Genes Dev. 28, 812-828. https://doi.org/10.1101/gad.234294.113

Mizuno, H., Matsumoto, T., Wu, J., 2018. Composition and Structure of Rice Centromeres and Telomeres, in: Sasaki, T., Ashikari, M. (Eds.), Rice Genomics, Genetics and Breeding. Springer Singapore, Singapore, pp. 37-52. https://doi.org/10.1007/978-981-10-7461-5_3

Moritoh, S., Eun, C.-H., Ono, A., Asao, H., Okano, Y., Yamaguchi, K., Shimatani, Z., Koizumi, A., Terada, R., 2012. Targeted disruption of an orthologue of DOMAINS REARRANGED METHYLASE 2, OsDRM2, impairs the growth of rice plants by abnormal DNA methylation: osdrm2 affects DNA 
methylation and development. Plant J. 71, 85-98. https://doi.org/10.1111/j.1365313X.2012.04974.x

Okada, T., Endo, M., Singh, M.B., Bhalla, P.L., 2005. Analysis of the histone H3 gene family in Arabidopsis and identification of the male-gamete-specific variant AtMGH3: Histone $\mathrm{H} 3$ gene family in Arabidopsis. Plant J. 44, 557-568. https://doi.org/10.1111/j.1365-313X.2005.02554.x

Papareddy, R.K., Páldi, K., Paulraj, S., Kao, P., Lutzmayer, S., Nodine, M.D., 2020. Chromatin regulates expression of small RNAs to help maintain transposon methylome homeostasis in Arabidopsis. Genome Biol. 21, 251. https://doi.org/10.1186/s13059-020-02163-4

Parent, J.-S., Cahn, J., Herridge, R.P., Grimanelli, D., Martienssen, R.A., 2021. Small RNAs guide histone methylation in Arabidopsis embryos. Genes Dev. 35, 841-846. https://doi.org/10.1101/gad.343871.120

Park, K., Kim, M.Y., Vickers, M., Park, J.-S., Hyun, Y., Okamoto, T., Zilberman, D., Fischer, R.L., Feng, X., Choi, Y., Scholten, S., 2016. DNA demethylation is initiated in the central cells of Arabidopsis and rice. Proc. Natl. Acad. Sci. 113, 15138-15143. https://doi.org/10.1073/pnas.1619047114

Pillot, M., Baroux, C., Arteaga Vazquez, M., Autran, D., Leblanc, O., Vielle-calzada, J.P., Grossniklaus, U., Grimanelli, D., 2010. Embryo and Endosperm Inherit Distinct Chromatin and Transcriptional States from the Female Gametes in Arabidopsis. Plant Cell 22, 307-320. https://doi.org/10.1105/tpc.109.071647

Quinlan, A.R., Hall, I.M., 2010. BEDTools: a flexible suite of utilities for comparing genomic features. Bioinformatics 26, 841-842. https://doi.org/10.1093/bioinformatics/btq033

Rajkumar, M.S., Gupta, K., Khemka, N.K., Garg, R., Jain, M., 2020. DNA methylation reprogramming during seed development and its functional relevance in seed size/weight determination in chickpea. Commun. Biol. 3, 340. https://doi.org/10.1038/s42003-020-1059-1

Rodrigues, J.A., Ruan, R., Nishimura, T., Sharma, M.K., Sharma, R., Ronald, P.C., Fischer, R.L., Zilberman, D., 2013a. Imprinted expression of genes and small RNA is associated with localized hypomethylation of the maternal genome in rice endosperm. Proc. Natl. Acad. Sci. 110, 79347939. https://doi.org/10.1073/pnas.1306164110

Rodrigues, J.A., Ruan, R., Nishimura, T., Sharma, M.K., Sharma, R., Ronald, P.C., Fischer, R.L., Zilberman, D., 2013b. Imprinted expression of genes and small RNA is associated with localized hypomethylation of the maternal genome in rice endosperm. Proc. Natl. Acad. Sci. 110, 79347939. https://doi.org/10.1073/pnas.1306164110

Saitou, M., Kagiwada, S., Kurimoto, K., 2012. Epigenetic reprogramming in mouse pre-implantation development and primordial germ cells. Development 139, 15-31. https://doi.org/10.1242/dev.050849

Satyaki, P.R. V, Gehring, M., 2019. Paternally Acting Canonical RNA-Directed DNA Methylation Pathway Genes Sensitize Arabidopsis Endosperm to Paternal. Plant Cell 31, 1563-1578. https://doi.org/10.1105/tpc.19.00047

Schmieder, R., Edwards, R., 2011. Quality control and preprocessing of metagenomic datasets. Bioinformatics 27, 863-864. https://doi.org/10.1093/bioinformatics/btr026

Schoft, V.K., Chumak, N., Mosiolek, M., Slusarz, L., Komnenovic, V., Brownfield, L., Twell, D., Kakutani, T., Tamaru, H., 2009. Induction of RNA-directed DNA methylation upon decondensation of constitutive heterochromatin. Sci. Rep. 10, 1015-1021. https://doi.org/10.1038/embor.2009.152

Scholten, S., Lörz, H., Kranz, E., 2002. Paternal mRNA and protein synthesis coincides with male chromatin decondensation in maize zygotes. Plant J. 32, 221-231. https://doi.org/10.1046/j.1365-313X.2002.01418.x 
1063

1064

1065

1066

1067

1068

1069

1070

1071

1072

1073

1074

1075

1076

1077

1078

1079

1080

1081

1082

1083

1084

1085

1086

1087

1088

1089

1090

1091

1092

1093

1094

1095

1096

1097

1098

1099

1100

1101

1102

1103

1104

1105

1106

1107

1108

1109
Searle, S.R., Speed, F.M., Milliken, G.A., 1980. Population Marginal Means in the Linear Model: An Alternative to Least Squares Means. Am. Stat. 34, 216-221. https://doi.org/10.1080/00031305.1980.10483031

Slotkin, R Keith, Vaughn, M., Borges, F., Feijo, A., Becker, D., Martienssen, R.A., 2009. Epigenetic Reprogramming and Small RNA Silencing of Transposable Elements in Pollen. Cell 461-472. https://doi.org/10.1016/j.cell.2008.12.038

Slotkin, R. Keith, Vaughn, M., Borges, F., Tanurdžić, M., Becker, J.D., Feijó, J.A., Martienssen, R.A., 2009. Epigenetic Reprogramming and Small RNA Silencing of Transposable Elements in Pollen. Cell 136, 461-472. https://doi.org/10.1016/j.cell.2008.12.038

Su, Z., Wang, N., Hou, Z., Li, B., Li, D., Liu, Y., Cai, H., Qin, Y., Chen, X., 2020. Regulation of Female Germline Specification via Small RNA Mobility in Arabidopsis. Plant Cell tpc.00126.2020. https://doi.org/10.1105/tpc.20.00126

Tadros, W., Lipshitz, H.D., 2009. The maternal-to-zygotic transition: a play in two acts. Development 136, 3033-3042. https://doi.org/10.1242/dev.033183

Tan, F., Lu, Y., Jiang, W., Zhang, R., Zhao, Y., Zhou, D., 2018. DDM1 Represses Noncoding RNA Expression and RNA-Directed DNA Methylation in Heterochromatin 177, 1187-1197. https://doi.org/10.1104/pp.18.00352

Tan, F., Zhou, C., Zhou, Q., Zhou, S., Yang, W., Zhao, Y., Li, G., 2016. Analysis of Chromatin Regulators Reveals Speci fi c Features of Rice DNA Methylation Pathways. Plant Physiol. 171, 2041-2054. https://doi.org/10.1104/pp.16.00393

Tang, W.W.C., Kobayashi, T., Irie, N., Dietmann, S., Surani, M.A., 2016. Specification and epigenetic programming of the human germ line. Nat. Rev. Genet. 17, 585-600.

https://doi.org/10.1038/nrg.2016.88

Thorvaldsdottir, H., Robinson, J.T., Mesirov, J.P., 2013. Integrative Genomics Viewer (IGV): highperformance genomics data visualization and exploration. Brief. Bioinform. 14, 178-192. https://doi.org/10.1093/bib/bbs017

Wang, G., Köhler, C., 2017. Epigenetic processes in flowering plant reproduction. J. Exp. Bot. erw486. https://doi.org/10.1093/jxb/erw486

Wang, Z., Butel, N., Santos-González, J., Borges, F., Yi, J., Martienssen, R.A., Martinez, G., Köhler, C., 2020. Polymerase IV Plays a Crucial Role in Pollen Development in Capsella. Plant Cell 32, 950966. https://doi.org/10.1105/tpc.19.00938

$\mathrm{Xu}$, Q., Xie, W., 2018. Epigenome in Early Mammalian Development: Inheritance, Reprogramming and Establishment. Trends Cell Biol. 28, 237-253. https://doi.org/10.1016/j.tcb.2017.10.008

Ye, R., Wang, W., Iki, T., Liu, C., Wu, Y., Ishikawa, M., Zhou, X., Qi, Y., 2012. Cytoplasmic Assembly and Selective Nuclear Import of Arabidopsis ARGONAUTE4/siRNA Complexes. Mol. Cell 46, 859-870. https://doi.org/10.1016/j.molcel.2012.04.013

Zemach, A., Kim, M.Y., Hsieh, P.-H., Coleman-Derr, D., Eshed-Williams, L., Thao, K., Harmer, S.L., Zilberman, D., 2013. The Arabidopsis Nucleosome Remodeler DDM1 Allows DNA Methyltransferases to Access H1-Containing Heterochromatin. Cell 153, 193-205. https://doi.org/10.1016/j.cell.2013.02.033

Zhao, P., Zhou, X., Shen, K., Liu, Z., Cheng, T., Liu, D., Cheng, Y., Peng, X., Sun, M., 2019. Two-Step Maternal-to-Zygotic Transition with Two-Phase Parental Genome Contributions. Dev. Cell 49, 882-893.e5. https://doi.org/10.1016/j.devcel.2019.04.016

Zhou, S., Li, X., Liu, Q., Zhao, Y., Jiang, W., Wu, A., Zhou, D.-X., 2021. DNA demethylases remodel DNA methylation in rice gametes and zygote and are required for reproduction. Mol. Plant S1674205221002185. https://doi.org/10.1016/j.molp.2021.06.006 
A Chr

$6 \quad 7$

$\begin{array}{lll}8 & 9 & 10\end{array}$

11

12

Centrom.

Genes

Gypsy

Seedling

Sperm

Egg

Zygote

Ovary (Ohr)

Ovary (9hr)

B

$$
\begin{aligned}
& \text { - ovary (0hr) }- \text { seedling }- \text { ovary (9hap) } \\
& - \text { egg } \quad \text { sperm }- \text { zygote }
\end{aligned}
$$
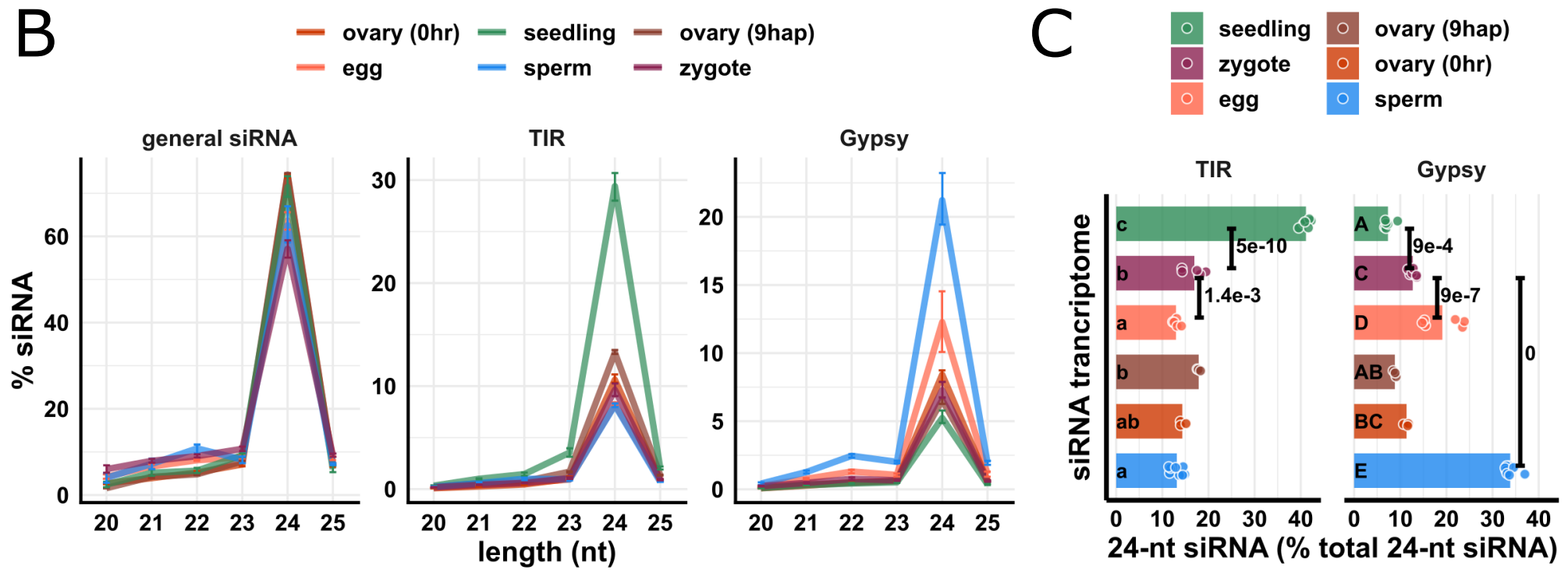

D
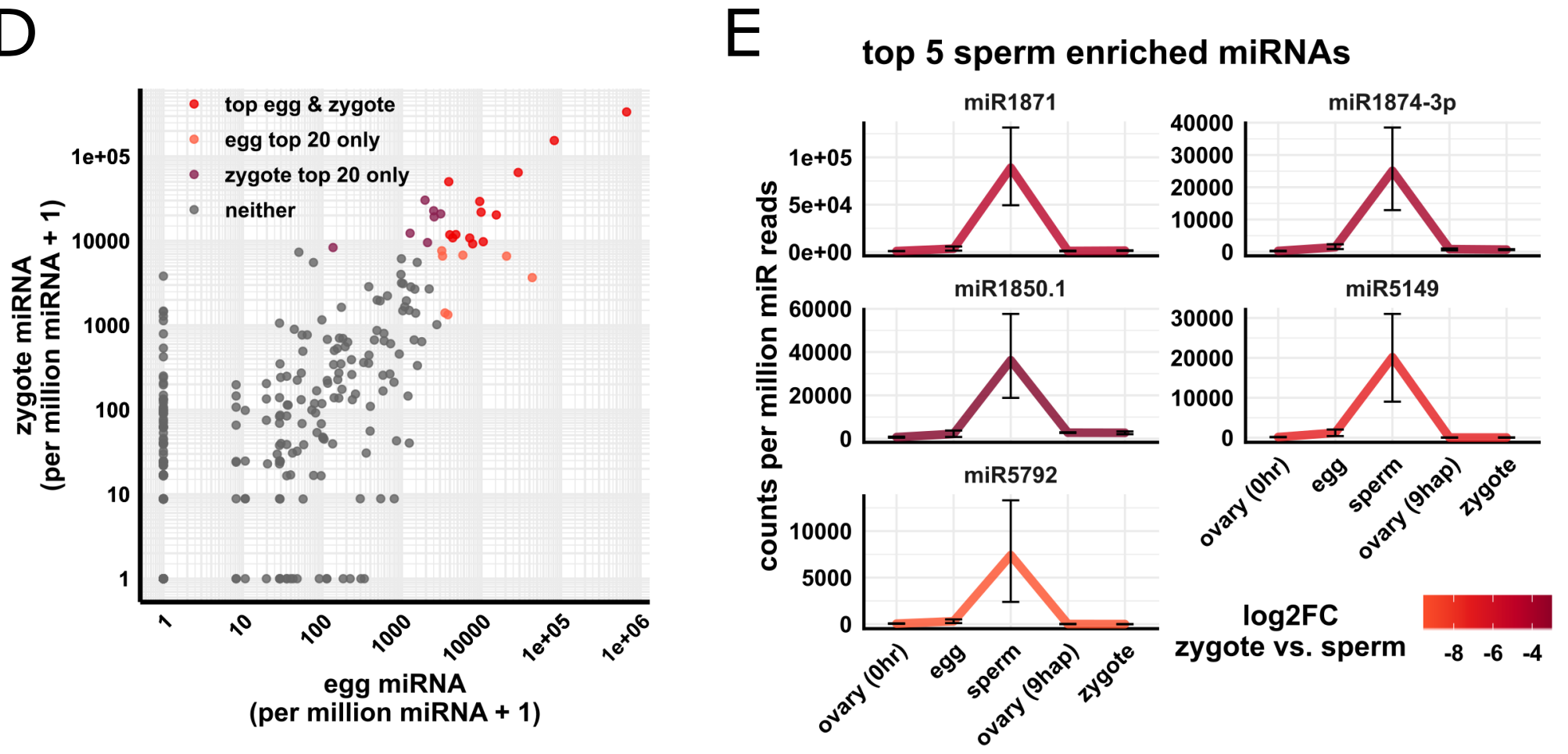
Fig 1: Overall pattern of zygote 24-nt siRNAs is similar to but not identical to egg cell.

(A) Heat map showing abundance of 24-nt siRNA across genome at 50-kb resolution. The first three tracks are centromeres [as defined by (Mizuno et al., 2018)], genes, and Gypsy retrotransposons.

(B) Length profiles of siRNAs. y-axis values are relative to total siRNA reads $(20-25-\mathrm{nt}$ siRNAs). TIR: terminal inverted repeat transposons, CACTA superfamily excluded. Gypsy: Gypsy retrotransposons. Error bars are 95\% confidence intervals for each cell type. miRNA and phasiRNA are not included in this analysis (Fig S1A).

(C) Quantification of TIR and Gypsy panels in (B). Each data point is an siRNA transcriptome. Bar heights are averages. $\mathrm{x}$-axis values are relative to total 24-nt siRNAs. Letter grouping ( $\alpha=0.05)$ and $\mathrm{P}$ values are based on Tukey tests.

(D) Scatter plot showing miRNA relative abundances in egg and zygote. Each data point is a miRNA. Axes are relative to per million miRNA reads and $\log 10$ transformed. 'top egg \& zygote' refers to intersection of the 20 highest abundant miRNAs in both egg and zygote.

(E) Top five sperm enriched miRNAs. Sperm enriched is classified as $>1000$ reads per million miRNA reads in sperm and $<500$ reads per million miRNA reads in egg. $y$-axis values are relative to per million miRNA reads. Color code reflects $\log 2 \mathrm{FC}$ values for zygote vs. sperm, and negative values indicate higher in sperm. Error bars are $95 \%$ confidence intervals for each cell type. See Fig S1D for additional examples.

Zygote and 9 hap ovary data are from this study, all other data from Li et al., (2020). 
A

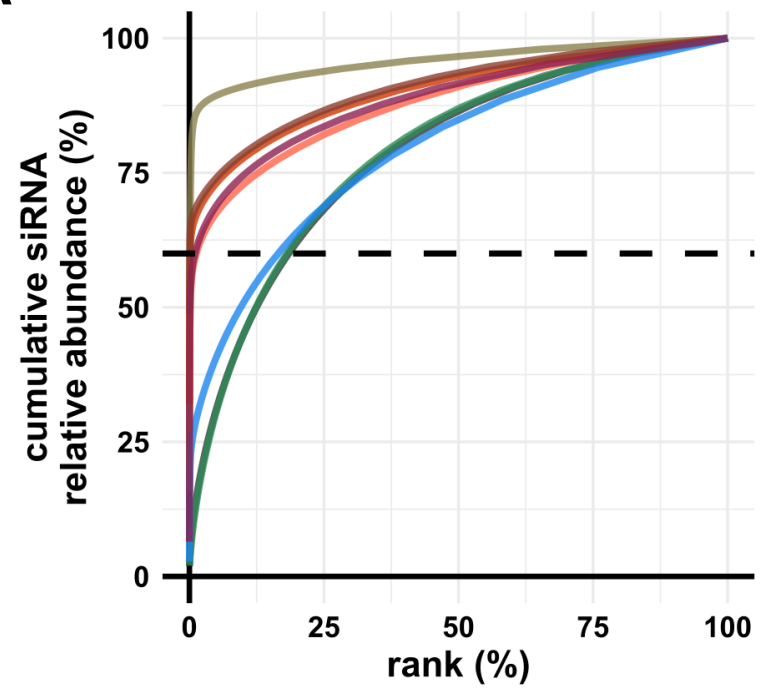

- ovary (0hr) - ovary (9hap)

- egg zygote

- seedling - embryo

- sperm endosperm
Pattern of siren loci across genome

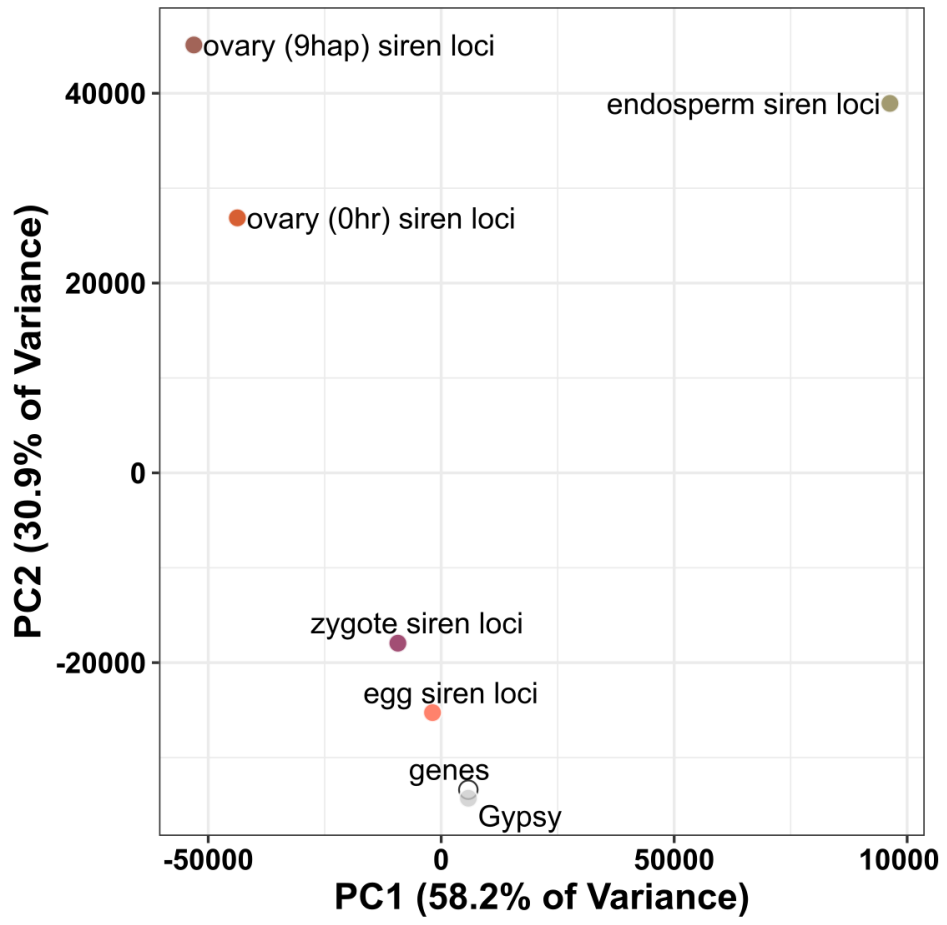

$B$

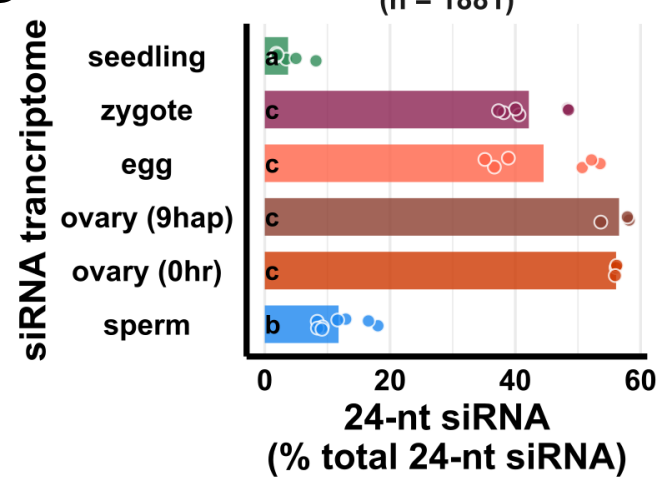

D

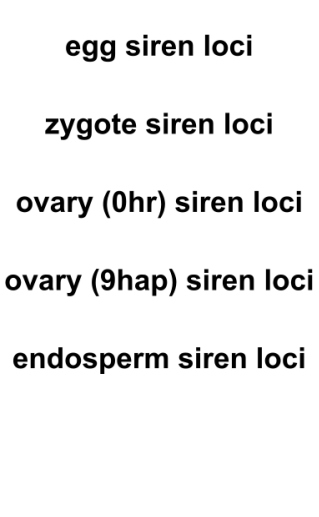

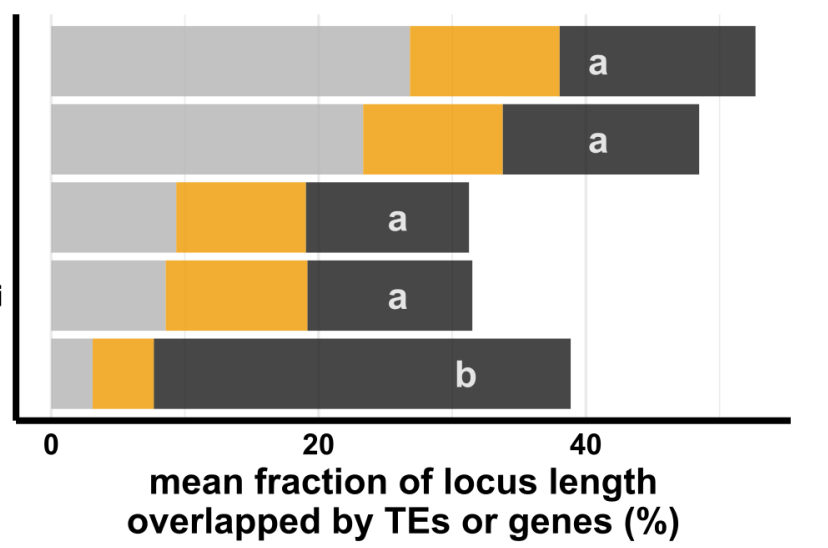

genes

TIR

Gypsy

\section{siRNA $\quad \boxminus$ ovary (0hr) $\boxminus$ seedling $\boxminus$ ovary (9hap) $\boxminus$ embryo transcriptome $\boxminus$ egg $\quad \boxminus$ sperm $\quad \boxminus$ zygote $\quad \boxminus$ endosperm}

$E$
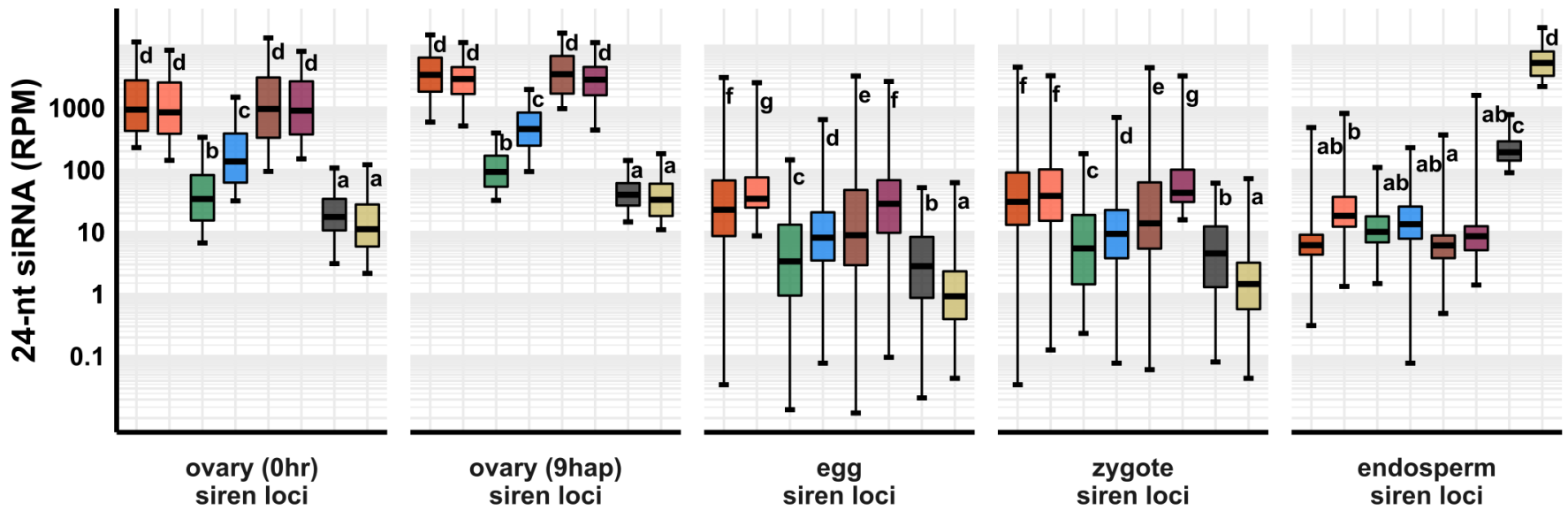
Fig 2: Zygote siren loci are similar to siren loci detected in ovary and egg cell and stably expressed between egg and zygote but dissimilar to siren loci detected in endosperm.

(A) $\mathrm{x}$-axis is the rank order of siRNA loci. siRNA loci with highest siRNA abundances are ranked first. $y$-axis is cumulative relative abundance of siRNA in all siRNA loci. Axis values are scaled between 0 and $100 \% .0 .1 \%$ of siRNA loci accounted for $60 \%$ of siRNA reads in all siRNA loci in endosperm and ovary. $1 \%$ of siRNA loci accounted for $60 \%$ of siRNA reads in all siRNA loci in egg and zygote.

(B) Bar plot showing relative abundances of 24-nt siRNA at egg siren loci. Each data point is an siRNA transcriptome. Bar heights are averages. $\mathrm{x}$-axis values are relative to total 24-nt siRNAs.

(C) Principal component plot for siren loci distribution across the genome. Distributions are evaluated at 50-kb resolution across the genome. Each data point is the distribution of a siren loci category.

(D) Stacked bar plots showing mean fraction of locus length overlapped by TEs or genes. TIR: terminal inverted repeat transposons, CACTA superfamily excluded. Gypsy: Gypsy retrotransposons.

(E) Boxplots showing 24-nt siRNA relative abundances across siren classes across cell types. Middle lines are median. Boxes span interquartile range. y-axis values are relative to per million total 24-nt siRNAs in each siRNA transcriptome. Whiskers span $2.5^{\text {th }}$ and $97.5^{\text {th }}$ percentiles.

Letter grouping $(\alpha=0.05)$ and $\mathrm{P}$ values are based on Tukey tests. Embryo and endosperm data from Rodrigues et al., (2013). Seedling, gametes, and pre-fertilization ovary data from Li et al., (2020). 


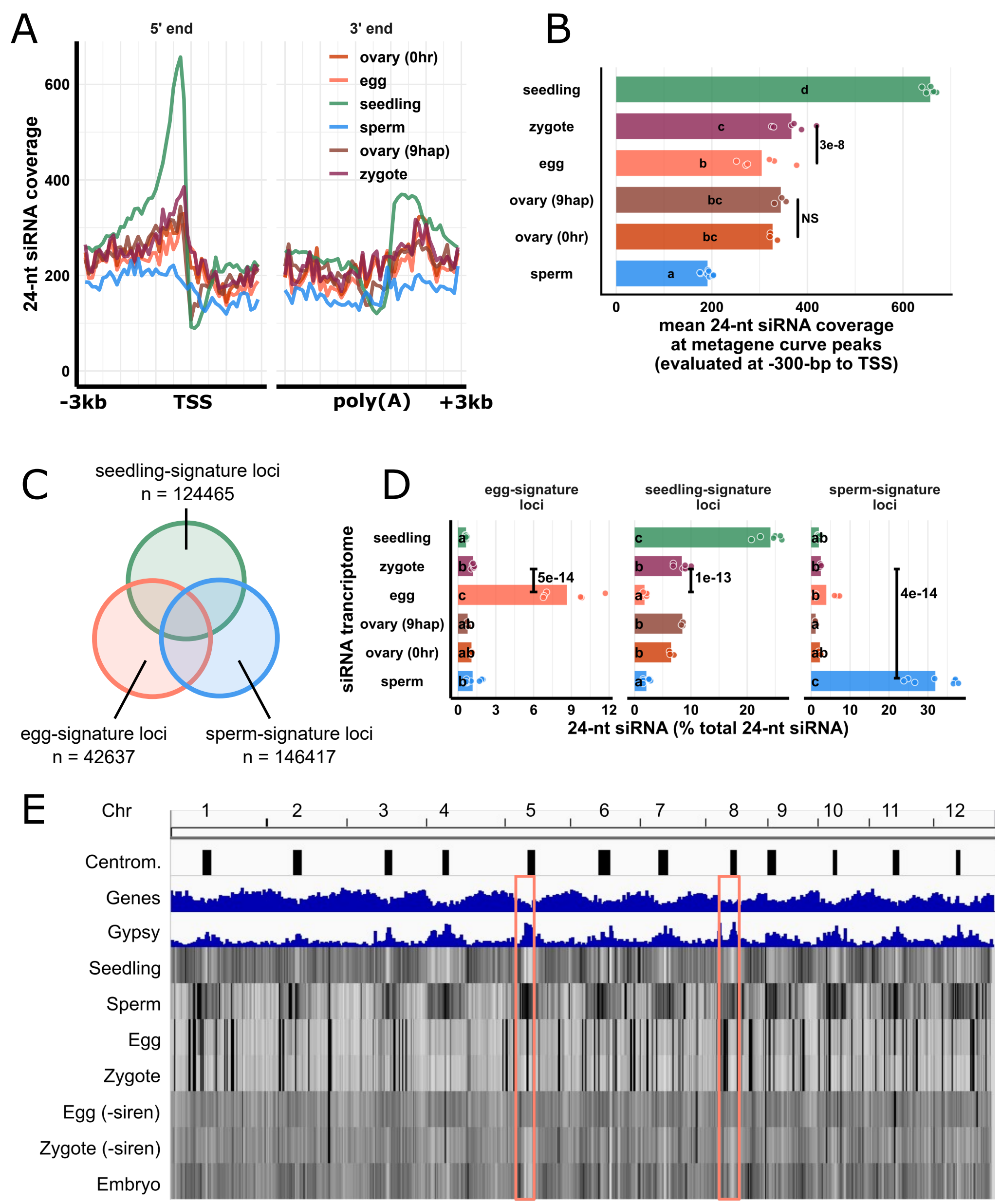


Fig 3: Changes in the zygote siRNA transcriptome are independent from the ovary after fertilization.

(A) Metagene coverage plot for 24-nt siRNAs. Coverage is measured over 100-bp intervals and normalized per 1000 24-nt siRNAs. Vertical grid lines are 500-bp intervals. TSS transcription start site, $\operatorname{poly}(\mathrm{A})$ polyadenylation site.

(B) Quantification of (A) at the interval from 300 to 200-bp upstream of TSS, corresponding to the peaks of metagene curves. Each data point is an siRNA transcriptome and bar heights are averages. $\mathrm{x}$-axis values are normalized per 1000 24-nt siRNAs.

(C) Venn diagram illustrating egg-signature loci (egg siRNA loci that do not overlap any seedling or sperm siRNA loci), seedling-signature loci (seedling siRNA loci that do not overlap any egg or sperm siRNA loci), and sperm-signature loci (sperm siRNA loci that do not overlap any egg or seedling siRNA loci). Sizes of overlap in Venn diagrams are not to scale.

(D) Bar plot showing relative abundances of 24-nt siRNA across siRNA loci categories defined in (C). The zygote siRNA transcriptome was not used to define these locus categories. Each data point is an siRNA transcriptome. Bar heights are averages. $\mathrm{X}$-axis values are normalized to total 24-nt siRNAs.

(E) Heat map showing abundance of 24-nt siRNA across genome at 50-kb resolution. The first three tracks are centromeres [as defined by (Mizuno et al., 2018)], genes, and Gypsy retrotransposons. '-siren' refers to siren siRNAs removed. Pink boxes highlight examples where egg and zygote are distinct.

Letter grouping $(\alpha=0.05)$, and $\mathrm{P}$ values are based on Tukey tests. Zygote and 9 hap ovary data are from this study, embryo (7-8 DAF) from Rodrigues et al. (2013), all other data from Li et al., (2020). 
A

$\begin{array}{cc}\text { E loci }- \text { Z loci } & \text { Z loci }-E \text { loci } \\ n=101841 & n=94591\end{array}$

B

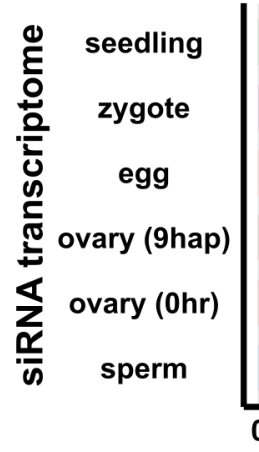

E loci - Z loci

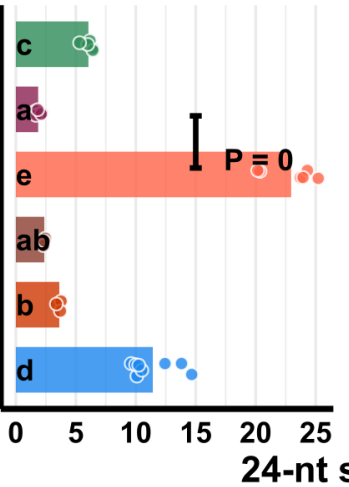

Z/E loci intersect
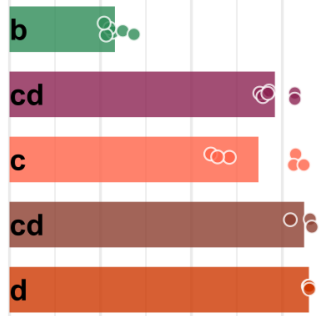

a 2000
Z/E loci intersect

$$
\mathrm{n}=42437
$$

Chromosome 8

Centrom.

Sperm siRNA loci

Genes

TIR

Gypsy

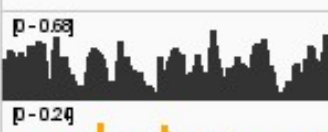

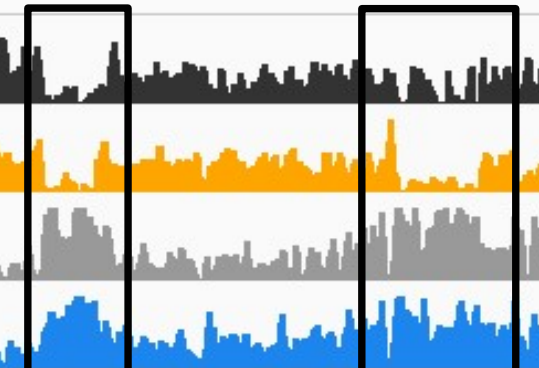

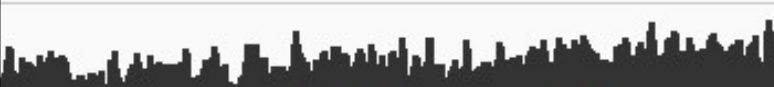
p-0.8q

Seedling siRNA loci

$$
\text { p-ing }
$$

p-029

DRM2 targets 然 p.om

Z loci - E loci

E loci - Z loci

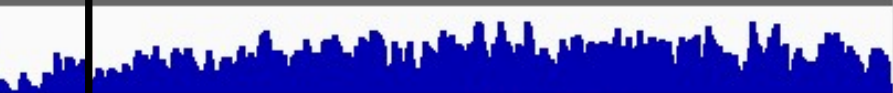

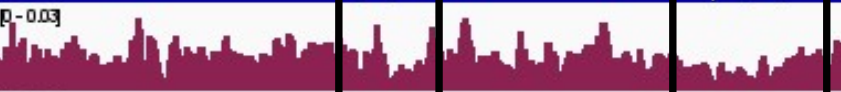

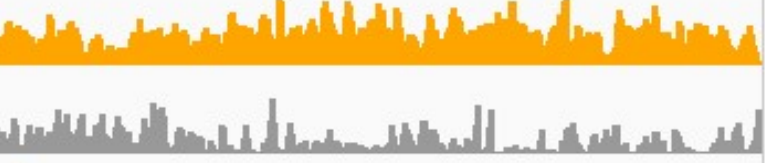

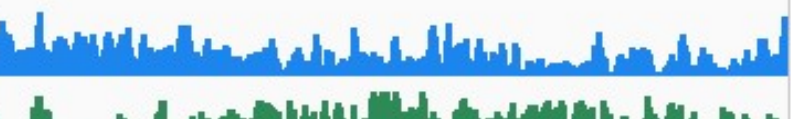

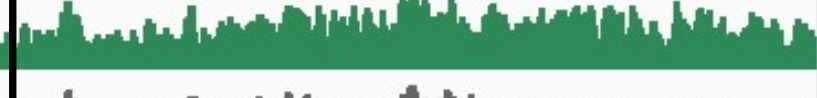
p-0.0q Wh

Pattern of siRNA loci distribution across the genome
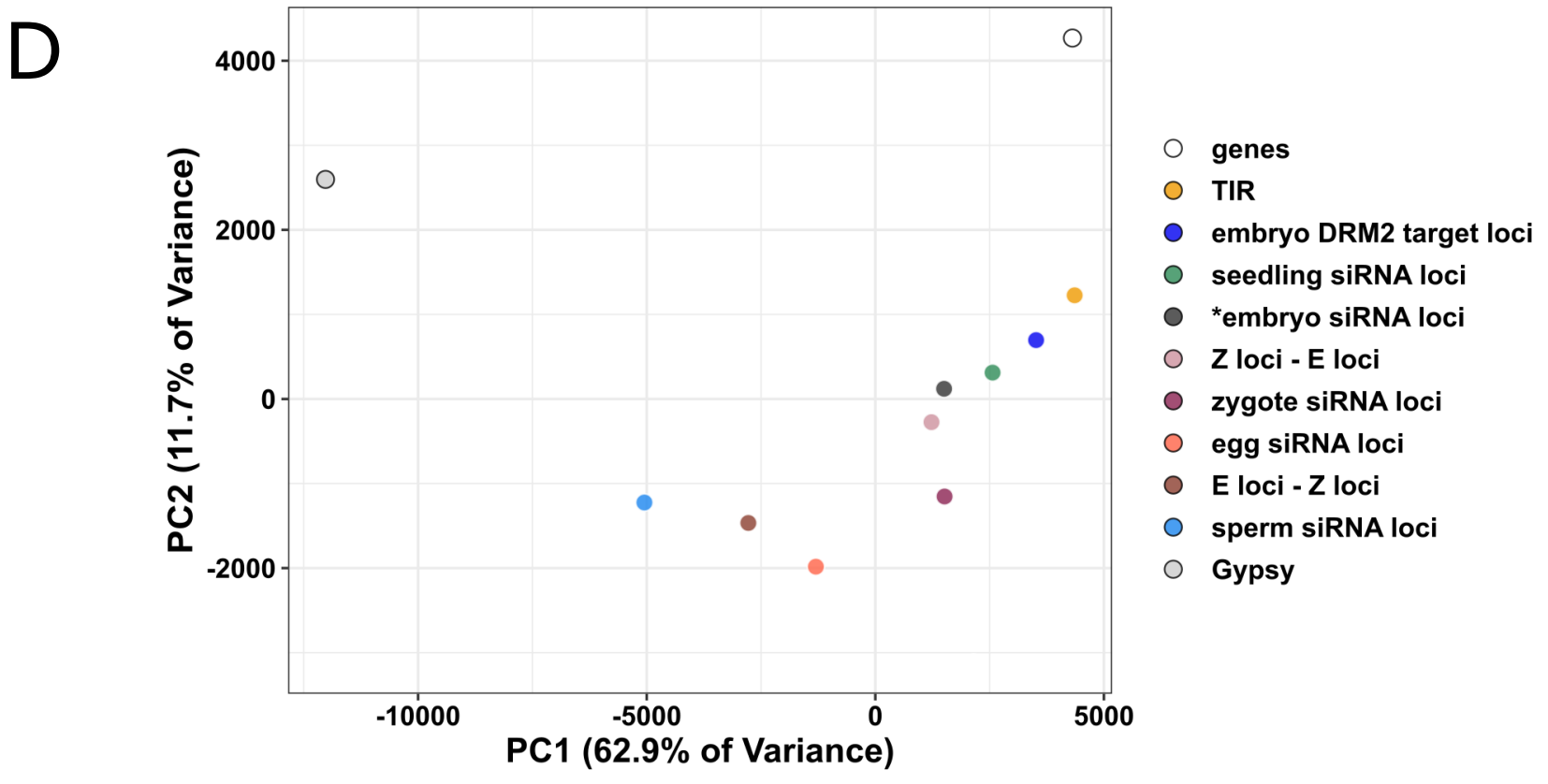


\section{Fig 4: Widespread newly detected siRNA loci in zygote.}

(A) Venn diagram illustrating E-Z loci (egg siRNA loci that do not overlap any zygote siRNA loci, E loci $\notin$ Z loci), Z-E loci (zygote siRNA loci that do not overlap any egg siRNA loci Z loci $\notin \mathrm{E}$ loci), and Z/E loci intersect (zygote siRNA loci that overlap egg siRNA loci, Z loci $\cap$ E loci). Sizes of overlap in Venn diagrams are not to scale.

(B) Quantification of 24-nt siRNA relative abundances for (A). Each data point is a siRNA transcriptome. Bar heights are averages. X-axis-values are relative to total 24-nt siRNA reads. Letter grouping $(\alpha=0.05)$, and P values are based on Tukey tests.

(C) Distribution of siRNA loci along a chromosome. Chromosome 8 is chosen because it is one of the chromosomes with a completed sequenced centromeric region (Mizuno et al., 2018). Centrom. Centromeric regions; TIR: terminal inverted repeat transposons, CACTA superfamily excluded. Gypsy: Gypsy retrotransposons. Black boxes highlight regions with abundant Gypsy retrotransposons and relative depletion of TIR, seedling siRNA loci, embryo siRNA loci, DRM2 targets, and Z-E loci.

(D) Principal component plot showing siRNA loci distribution across the genome.

Distributions are evaluated at $50-\mathrm{kb}$ resolution across the genome. Each data point is the distribution of a loci category.

Zygote and 9 hap ovary data are from this study, all other data from Li et al., (2020). 
egg siRNA loci zygote siRNA loci

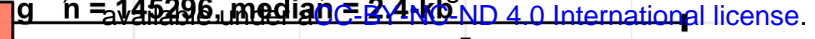
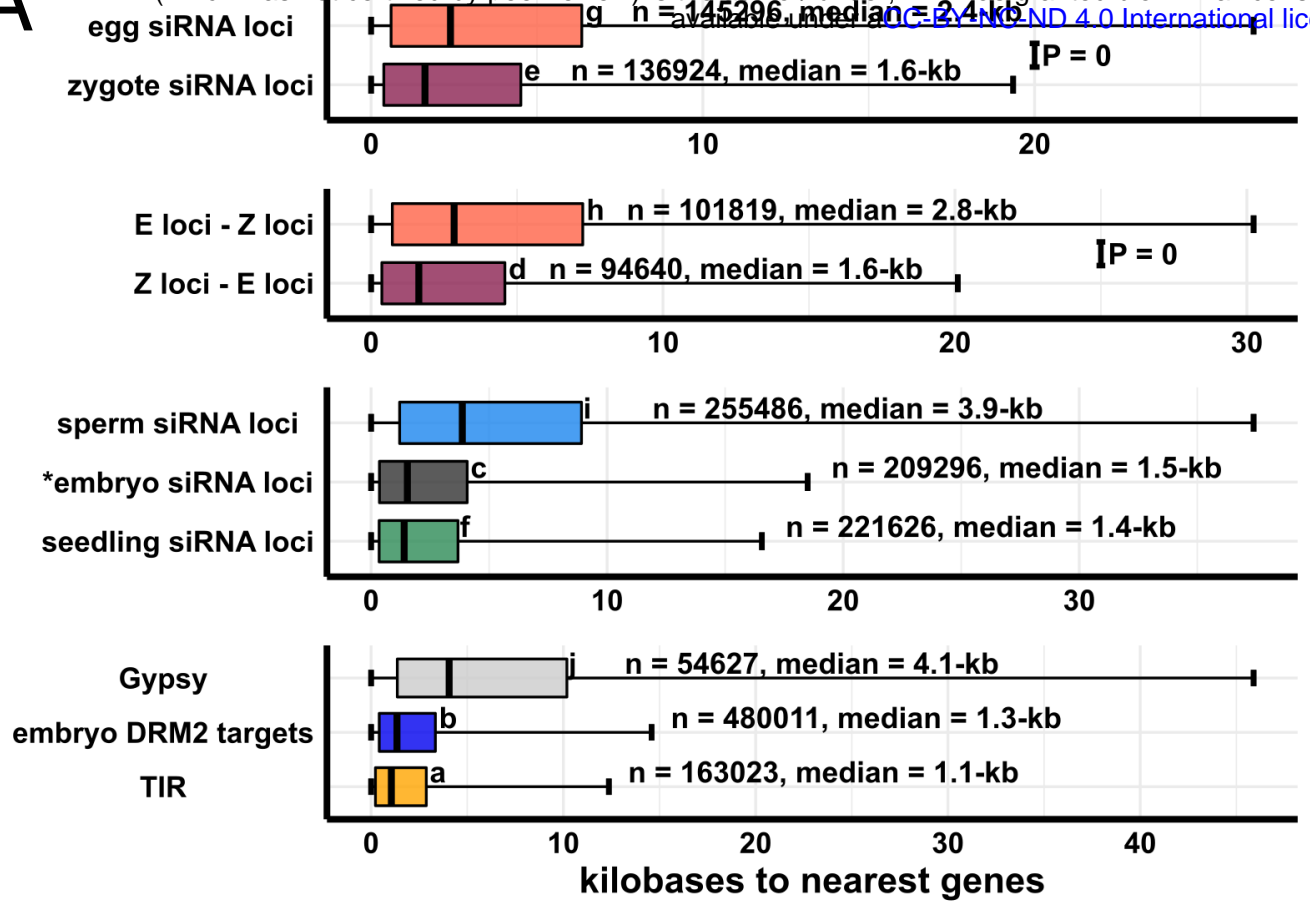

B

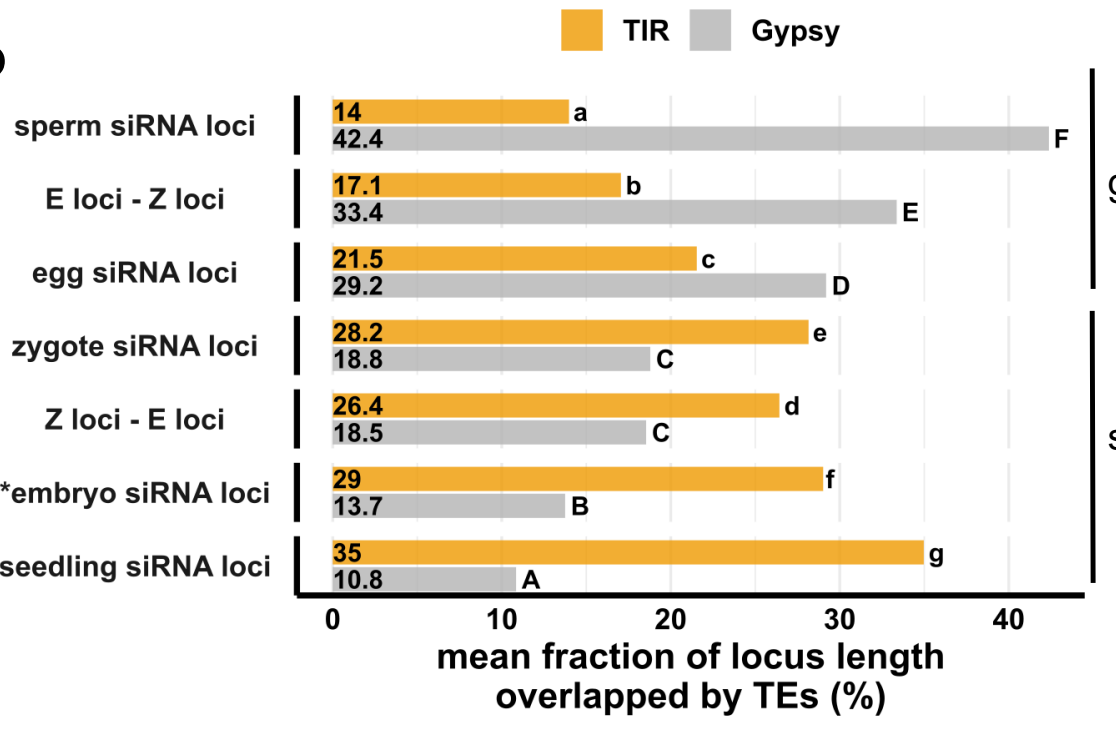

gametes

sporophytes

C

\begin{tabular}{|c|c|}
\hline seedling siRNA loci & ]$^{a}$ \\
\hline zygote siRNA loci & $a b c$ \\
\hline *embryo siRNA loci & b \\
\hline Z loci - E loci & hc \\
\hline egg siRNA loci & \\
\hline E loci - Z loci & bcd \\
\hline sperm siRNA loci & \\
\hline
\end{tabular}

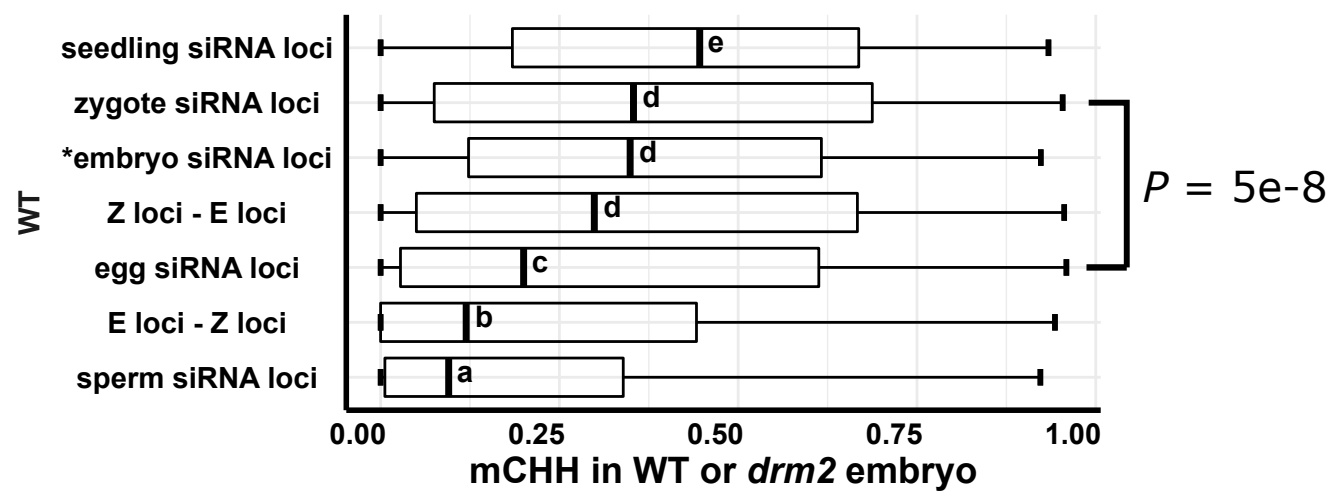


Fig 5: Newly detected siRNA loci in zygote reset to the canonical siRNA profile and predict CHH methylation in embryo in an RdDM-dependent manner.

(A) Boxplots showing distance of siRNA loci to nearest genes. Middle lines are median. Boxes span interquartile range. Whiskers $\operatorname{span} 2.5^{\text {th }}$ and $97.5^{\text {th }}$ percentiles.

(B) Bar plots showing mean locus length overlapped by TIR or Gypsy transposons across siRNA loci categories. Statistical comparisons are made across siRNA loci categories within a TE superfamily.

(C) Boxplots showing $\mathrm{CHH}$ methylation level in mature wildtype and $d r m 2$ mutant embryos. Middle lines are median. Boxes span interquartile range. Whiskers span $2.5^{\text {th }}$ and $97.5^{\text {th }}$ percentiles. E-Z loci: $n=101,841$, Z-E loci: $n=94,591$ (69\% of all zygote siRNA loci). Letter groupings $(\alpha=0.05)$ and $\mathrm{P}$ values are based on Tukey tests. *Embryo siRNA data from Rodrigues et al., (2013), which was based on a single replicate. Seedling, gametes, and prefertilization ovary data from Li et al., (2020). 